\title{
Systems and synthetic biology to elucidate secondary metabolite biosynthetic gene clusters encoded in Streptomyces genomes
}

Lee, Namil; Hwang, Soonkyu; Kim, Woori; Lee, Yongjae; Kim, Ji Hun; Cho, Suhyung; Kim, Hyun Uk; Yoon, Yeo Joon; Oh, Min-Kyu; Palsson, Bernhard O

Total number of authors:

11

Published in:

Natural Product Reports

Link to article, DOI:

10.1039/d0np00071j

Publication date:

2021

Document Version

Peer reviewed version

Link back to DTU Orbit

Citation (APA):

Lee, N., Hwang, S., Kim, W., Lee, Y., Kim, J. H., Cho, S., Kim, H. U., Yoon, Y. J., Oh, M-K., Palsson, B. O., \& Cho, B-K. (2021). Systems and synthetic biology to elucidate secondary metabolite biosynthetic gene clusters encoded in Streptomyces genomes. Natural Product Reports, 38, 1330-1361.

https://doi.org/10.1039/d0np00071j

\section{General rights}

Copyright and moral rights for the publications made accessible in the public portal are retained by the authors and/or other copyright owners and it is a condition of accessing publications that users recognise and abide by the legal requirements associated with these rights.

- Users may download and print one copy of any publication from the public portal for the purpose of private study or research.

- You may not further distribute the material or use it for any profit-making activity or commercial gain

- You may freely distribute the URL identifying the publication in the public portal 
Received 00th January 20xx, Accepted 00th January 20xx DOI: $10.1039 / x 0 x \times 00000 x$

\title{
Systems and synthetic biology to elucidate secondary metabolite biosynthetic gene clusters encoded in Streptomyces genomes
}

\begin{abstract}
Namil Lee, \#ab Soonkyu Hwang, ${ }^{\text {ab }}$ Woori Kim, ${ }^{\text {ab }}$ Yongjae Lee, ${ }^{\text {ab }}$ Ji Hun Kim, ${ }^{\text {ab }}$ Suhyung Cho, ${ }^{\text {ab }}$ Hyun Uk Kim, ${ }^{c}$ Yeo Joon Yoon, ${ }^{* d}$ Min-Kyu Oh, ${ }^{*}$ Bernhard O. Palsson ${ }^{*}$ fgh and Byung-Kwan Cho ${ }^{*}$ abh

Over the last few decades, Streptomyces have been extensively investigated for their ability to produce diverse bioactive secondary metabolites. Recent advances in Streptomyces research have been largely supported by improvements in highthroughput technology 'omics.' From genomics, numerous secondary metabolite biosynthetic gene clusters were predicted, increasing their genomic potential for novel bioactive compound discovery. Additional omics, including transcriptomics, translatomics, interactomics, proteomics and metabolomics, have been applied to obtain a system-level understanding spanning entire bioprocesses of Streptomyces, revealing highly interconnected and multi-layered regulatory networks for secondary metabolism. The comprehensive understanding derived from this systematic information accelerates the rational engineering of Streptomyces to enhance secondary metabolite production, integrated with the exploitation of the highly efficient 'Design-Build-Test-Learn' cycle in synthetic biology. In this review, we describe the current status of omics applications in Streptomyces research to better understand the organism and exploit its genetic potential for higher production of valuable secondary metabolites and novel secondary metabolite discovery.
\end{abstract}

\section{Contents}

1. Introduction

2. Genomics: Establishment of fundamental genetic resources

2.1. Publicly available Streptomyces genomes

2.2. Identification of smBGCs encoded in Streptomyces genomes

2.3. Characterisation of natural products synthesised by the smBGCs

2.4. Understanding gene function and fitness using functional genomics

3. Transcriptomics: Elucidation of transcriptional regulation of secondary metabolism

\footnotetext{
a Department of Biological Sciences, Korea Advanced Institute of Science and Technology, Daejeon 34141, Republic of Korea

${ }^{\mathrm{b}}$ Innovative Biomaterials Centre, KI for the BioCentury, Korea Advanced Institute of Science and Technology, Daejeon 34141, Republic of Korea

c Department of Chemical and Biomolecular Engineering, Korea Advanced Institute of Science and Technology, Daejeon 34141, Republic of Korea

${ }^{d}$ College of Pharmacy, Seoul National University, Seoul 08826, Republic of Korea

e Department of Chemical and Biological Engineering, Korea University, Seoul 02841 Republic of Korea

${ }^{f}$ Department of Bioengineering, University of California San Diego, La Jolla, CA, 92093, USA

g Department of Pediatrics, University of California San Diego, La Jolla, CA, 92093, USA

h Novo Nordisk Foundation Centre for Biosustainability, Technical University of Denmark, Lyngby, 2800, Denmark

*Corresponding authors: Y.J.Y. (yeojoonyoon@snu.ac.kr), M.-K.O. (mkoh@korea.ac.kr), B.O.P. (bpalsson@ucsd.edu) and B.-K.C. (bcho@kaist.ac.kr)

\#These authors contributed equally to this work.

*These corresponding authors contributed equally to this work.

Electronic Supplementary Information (ESI) available: [details of any supplementary information available should be included here]. See DOI: 10.1039/x0xx00000x
}

3.1 Time-course transcriptome profiling and genetic development

3.2 Comparative transcriptomics for understanding the regulation of secondary metabolism

3.3 Determination of the regulatory elements encoded in Streptomyces genomes

4. Interactomics: Construction of transcriptional regulatory networks

4.1 Sigma factors

4.2 Transcription factors

5. Translatomics: Elucidation of translational regulation of secondary metabolism

6. Proteomics: Understanding secondary metabolism and discovery of natural products in Streptomyces

6.1 Comparative proteomics for understanding and identifying potential targets for enhancing SM production

6.2 Targeted proteomics for the discovery of novel SM and linking to smBGCs

7. Metabolomics: Understanding dynamic metabolite profiles from primary to secondary metabolism and identifying novel SMs

7.1 Understanding and identifying potential targets for enhancing SM production

7.2 Discovery of novel SMs and linking to smBGCs

8. Concluding remarks

9. Author contributions

10. Conflicts of interest

11. Acknowledgements

12. References 


\section{Introduction}

Actinobacteria are the representative sources of promising natural products, with their secondary metabolites (SMs) exhibiting a broad range of pharmaceutical bioactivities, such as antimicrobial, antifungal, anticancer and immunosuppressive activities. ${ }^{1}$ In particular, Streptomyces, the largest genus of actinobacteria, has received great attention as an industrial producer of natural products. More than 1,000 Streptomyces

Streptomyces
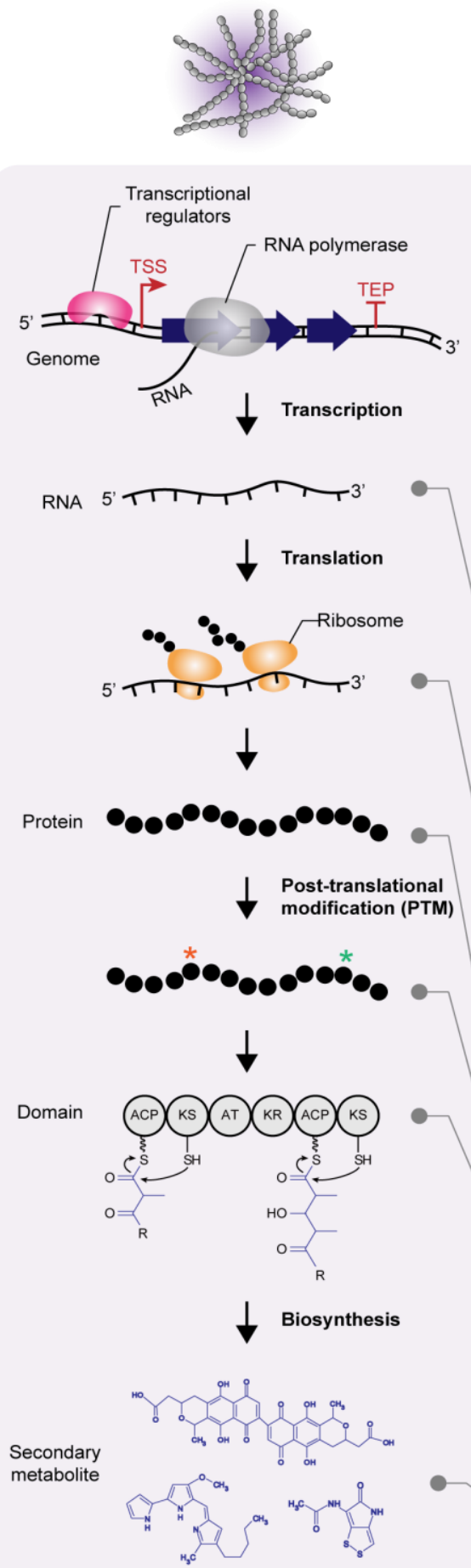

Fig. 1 Schematic overview of systems and synthetic biology on Streptomyces

Genomics

GEM

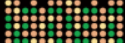

Transcriptomics

Translatomics strains have been reported in the NCBI taxonomy database and individual strains have the potential to produce distinct SMs; therefore, Streptomyces is an immeasurably rich source for novel SM discovery.

Biosynthetic pathways of SMs are encoded in large secondary metabolite biosynthetic gene clusters (smBGCs) in the genome, which include genes for biosynthesis, modification, transportation, regulation and resistance. ${ }^{2}$ On average, one
Systems biology

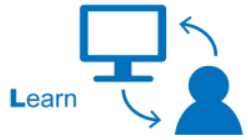

Genome mining
Synthetic biology
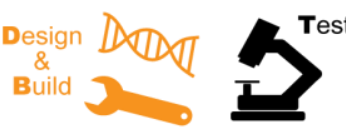

smBGC characterization
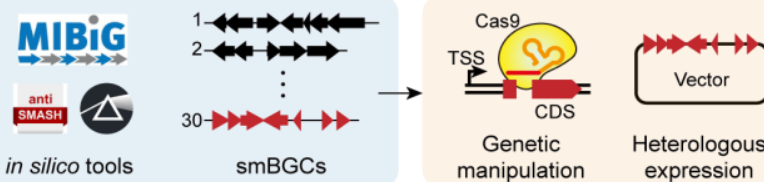

in silico tools

manipulation

expression

Understanding metabolic network
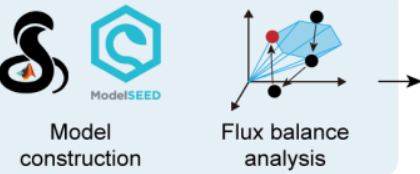

Bottleneck determination

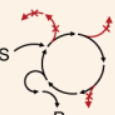

Knockout

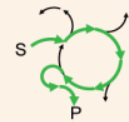

target

Overexpression

Transcriptional regulon identification
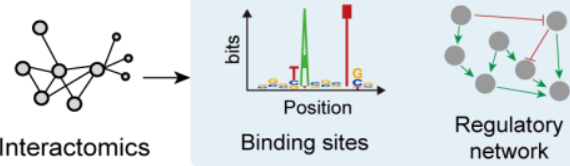

Regulon based engineering

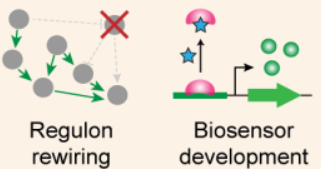

Understanding transcriptional regulation

Genetic part development
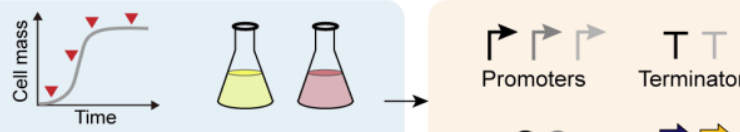

Promoters Terminators

Time-course

Comparative transcriptomics

$\Rightarrow \Rightarrow$

Understanding translational regulation

Altering tanslational efficiency

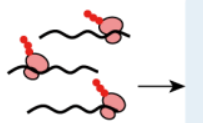

High ob

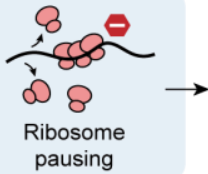

$\overrightarrow{\mathrm{C}} \rightarrow$ 5. UTR \&RBS

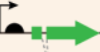

Engineering Engineering initiation step elongation step

Understanding direct functional units

Biosynthetic machinery engineering
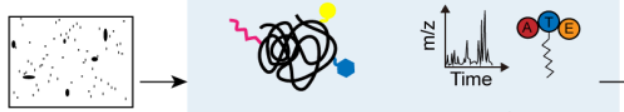

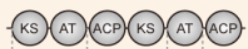

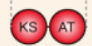

Domain \& module engineering for retro-biosynthesis

identification characterization

Key and novel metabolites identification
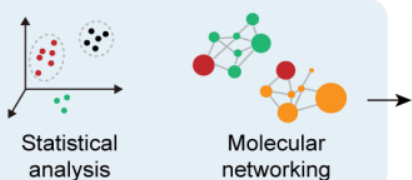

Engineered strain validation
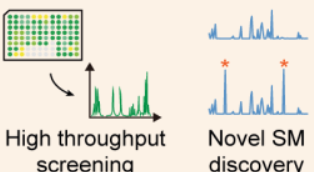

discovery 
Streptomyces strain possesses more than 30 smBGCs in its genome. ${ }^{3}$ However, their genetic potential has been poorly studied because many smBGCs are not activated under standard culture conditions. Although secondary metabolism is generally nonessential for cell maintenance, it is inseparably linked with primary metabolism; thus, Streptomyces controls secondary metabolism through multi- layered regulation across different levels of the biological information flow, such as transcription and translation. This complex and interlaced regulatory network is usually triggered by various biotic and abiotic stresses and ultimately induces SM production. Therefore, identifying novel SMs, understanding the unexplored genetic regulation and engineering the production of SMs are the top priorities for Streptomyces research.

Recently, rapid advances in next-generation sequencing (NGS) technology have enabled a systematic understanding of organisms by simultaneously analysing several layers of biological information flow. NGS techniques have facilitated quantitative tracking of changes in the genome, transcriptome, and translatome, which not only close the gap between genotype and phenotype but also enable the analysis of various regulations that orchestrate the cellular system by integrating disparate data (Fig. 1). ${ }^{4}$ Moreover, the recent advent of analytical technologies, including high-resolution mass spectrometry (MS) and nuclear magnetic resonance (NMR) spectroscopy, has enabled the quantitative measurement of the dynamic proteome and metabolome, which directly represent the cellular phenotypes derived from complex regulatory systems encoded in the genome (Fig. 1).5,6 The proteome and metabolome also shed light on the understanding of posttranslational regulation, including chemical modifications of proteins and diverse derivatisation of SMs in response to rapid environmental perturbations. In this context, we discuss recent progress in systems biology to understand the secondary metabolism of Streptomyces and discover novel bioactive SMs. Furthermore, for rational engineering of Streptomyces, the design (D), build (B), test (T), and learn (L) steps (the DBTL cycle

A
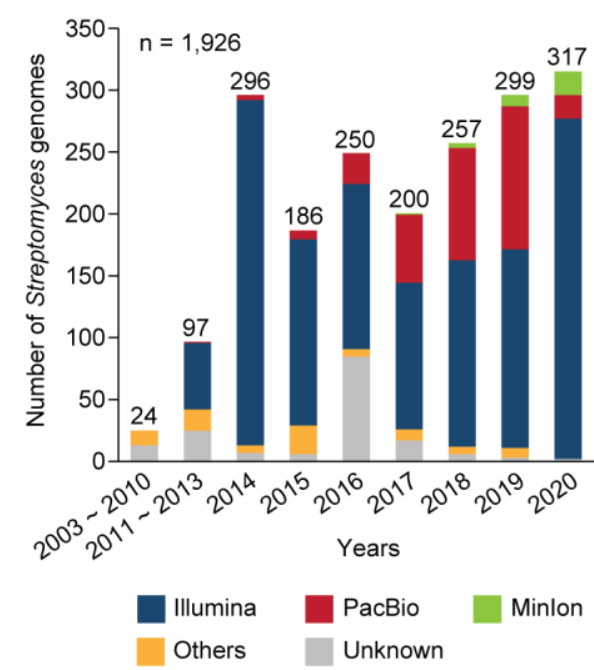

B

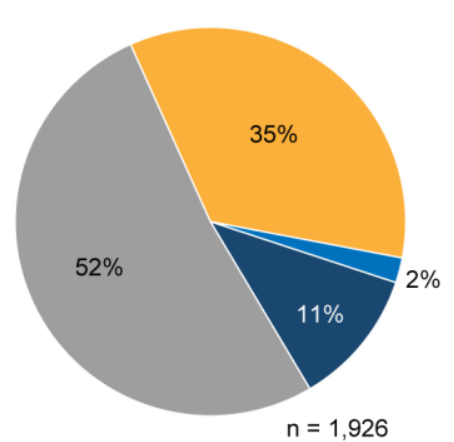

Complete

Chromosome

Scaffold

Contig of synthetic biology) are undoubtedly required (Fig. 1). Lastly, we highlight current limitations and suggest future perspectives on characterizing and engineering Streptomyces.

\section{Genomics: Establishment of fundamental genetic resources}

In the pre-genomic era, the identification of smBGCs relied on the molecular cloning of large segments of Streptomyces genomes (cosmid or fosmid library). ${ }^{7}$ Based on the highly conserved sequences of catalytic domains of biosynthetic and tailoring enzymes in smBGCs, DNA hybridisation has been actively used to mine novel smBGCs from newly isolated Streptomyces strains. ${ }^{8}$ This conventional approach entered a new phase with the development of genome sequencing methods and in silico smBGC mining tools, which enabled the screening of novel smBGCs at a tremendous rate.

\subsection{Publicly available Streptomyces genomes}

Since the genome sequences of $S$. coelicolor $A 3(2)^{9}$ and $S$. avermitilis ${ }^{10}$ were reported in 2003 , a considerable number of Streptomyces genomes have been sequenced. ${ }^{3,11}$ Since 2013, with the development of NGS methods, hundreds of Streptomyces genomes have been deposited in the public databases every year (Fig. 2A). Among the NGS platforms, the Illumina platform has been utilised the most, and the applications of long-read sequencing platforms, such as PacBio and Minlon (Oxford Nanopore), are gradually increasing (Fig. 2A). Despite the rapid accumulation of genome sequence data, many low-quality data are currently included in public databases. Thus, we sought to analyse the data quality of Streptomyces genome sequencing projects based on 'genome quality' and 'genome completeness', which indicate the amount of ambiguous sequences and coverage level of the reported genome sequence over the actual genome, respectively.

According to the RefSeq database, a total of 1,926 Streptomyces genomes were deposited as of July 1, 2020.

Fig. 2 Current status of Streptomyces genome projects. (A) Number of annually reported Streptomyces genomes from 2003 to 2020 . (B) Genome assembly level of 1,926 Streptomyces genomes. (C) Genome assembly level according to sequencing techniques. 
Among the genomes, $223(11 \%)$ are 'complete level' genomes, which are gapless or contain $<10$ ambiguous sequences. In addition, 41 genomes (2\%) are 'chromosome level', which are complete chromosome sequences, but include gaps comprising many ambiguous sequences. For example, the genome of Streptomyces sp. CB01881 (GCA_002953255.1) has 2.4 million ambiguous sequences, comprising $22 \%$ of the chromosome. The remaining 1,662 genomes are 'scaffold level' (35\%) or 'contig level' (52\%), which comprises numerous contigs, the shortest unit of genome assembly or scaffolds created by connecting contigs (Fig. 2B). These imperfectly assembled genomes are often inappropriate for further analysis, including coding sequence prediction and functional annotation of genes. ${ }^{11}$ The high proportion of low-quality Streptomyces genomes is due to the difficulty in sequencing and assembling when dealing with the high $\mathrm{G} / \mathrm{C}$ contents or repeated regions in Streptomyces genome.

To secure high-quality Streptomyces genomes, long-read sequencing methods are superior to short-read sequencing methods. Indeed, among the Streptomyces genomes sequenced using long-read sequencing methods, such as PacBio or Minlon platform, $47 \%$ and $82 \%$ of the genomes were assembled into single chromosomes, respectively, whereas only $3 \%$ of the genomes were assembled into single chromosomes when utilising the Illumina platform (Fig. 2C). Occasionally, assembled contigs based on short sequencing reads are not always included in the assembled contigs obtained from long sequencing reads; ${ }^{12}$ thus, reconciling short- and long-read sequencing methods is the most appropriate procedure to secure high-quality Streptomyces genomes by complementing the shortcomings of each method. ${ }^{11}$

A

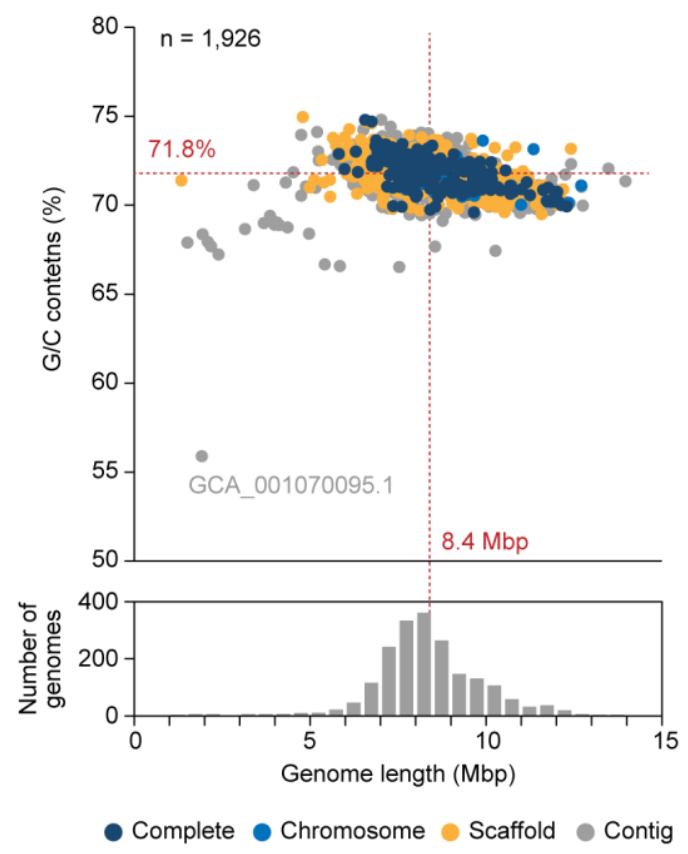

Streptomyces genomes have several unique features compared with other bacteria. First, Streptomyces is known to possess a large genome with high $\mathrm{G} / \mathrm{C}$ content. The average length of the 1,926 currently available Streptomyces genome sequences is $8.4 \mathrm{Mbp}$ and the average $\mathrm{G} / \mathrm{C}$ content is $71.8 \%$ (Fig. $3 \mathrm{~A})$. The large Streptomyces genomes encode approximately 7,000 protein-coding genes related to the distinct features of Streptomyces, including complex developmental cycles and secondary metabolism. The high $\mathrm{G} / \mathrm{C}$ content of the bacterial genome is known to correlate with a higher optimal growth temperature, ${ }^{13}$ but the reason for the high $\mathrm{G} / \mathrm{C}$ content of Streptomyces genomes is not yet completely understood. It is believed that high $\mathrm{G} / \mathrm{C}$ content will help to keep the large genome stable, ${ }^{13}$ prevent UV light damage, ${ }^{14}$ control smBGC expression with codon usage bias, ${ }^{15}$ and increase the chance of homologous recombination. ${ }^{16}$ In the case of contig level Streptomyces genomes with low G/C content, it is suspected that the initial sequencing materials were contaminated, interfering with the correct assembly of genomes (Fig. 3A). For instance, the G/C content of the S. cattleya 1093_SCAT (GCA_001070095.1) genome, comprising a total of 27 contigs, was $55.9 \%$. Despite being classified as the same species, the G/C content of the $S$. cattleya NRRL 8057 genome (GCA_000237305.1) was 73\%, suggesting that a large portion of the S. cattleya 1093_CAT genome is derived from other organisms.

Second, Streptomyces genomes form uniquely linear chromosomes. In particular, both arm regions are highly unstable and often cause large deletions, DNA duplications, and horizontal gene transfers, leading to variable phenotypic changes, allowing Streptomyces to adjust in ever-changing
B

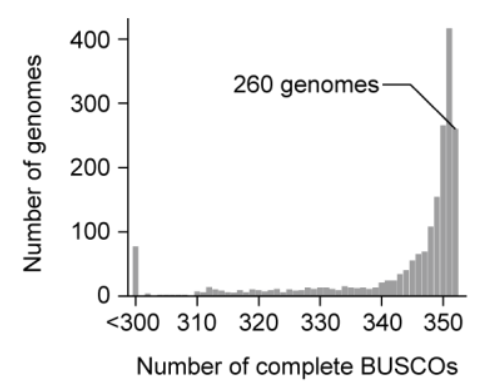

D

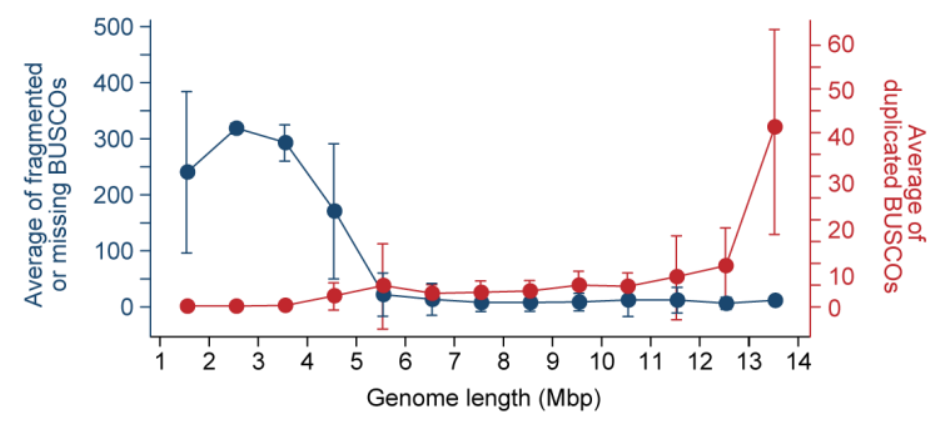

Fig. 3 Feature and completeness of 1,926 Streptomyces genomes. (A) Genome length and G/C content of 1,926 Streptomyces genomes. The red dotted lines represent the average value. (B) Assessing completeness of 1,926 Streptomyces genomes using BUSCO. (C) Relationship between genome assembly level and genome completeness. (D) Comparison of genome completeness according to length of Streptomyces genomes. 
environments. ${ }^{17,18}$ Compared to circular genomes of other bacteria, the linear genomes of Streptomyces are difficult to measure with genome sequencing. Alternatively, measuring gene space completeness indirectly predicts the completeness of Streptomyces genomes. Benchmarking Universal Single-Copy Orthologs (BUSCO) is a representative tool to measure gene space completeness of genomes by assessing the presence of conserved gene sets across the genus, which should exist as a single copy in genomes. ${ }^{19}$ Although a previous BUSCO analysis of 653 Streptomyces genomes concluded that $26 \%$ of the genomes have low completeness, ${ }^{20}$ the analysis was performed using only 40 BUSCO markers. Currently, the number of BUSCO markers for Streptomyces has increased to 352. In this regard, to evaluate gene space completeness of Streptomyces genomes, we performed a BUSCO analysis against the publicly available Streptomyces genomes. Among them, only 260 genomes (13.5\%) contained none of the fragmented or missisng BUSCOs, implying that the genomes were completely assembled (Fig. 3B). Scaffold and contig level genomes contained a significant amount of fragmented or missing BUSCOs compared to the complete and chromosome level genomes, meaning that some regions may be missing from the genomes (Fig. 3C). The number of fragmented or missing BUSCOs was drastically increased when the genome size was smaller than $6 \mathrm{Mbp}$. Nevertheless, a large genome size does not always correspond to the level of completeness of a genome. For example, Streptomyces genomes larger than $11 \mathrm{Mbp}$ contained numerous duplicated BUSCOs, which may represent the presence of repeat regions in the genome or multiple incorporations of the same region during genome assembly (Fig. 3D). Accordingly, 6-11 Mbp is considered to be the most reasonable genome size for Streptomyces.

Taken together, securing high-quality and complete Streptomyces genome sequences is essential for precise gene prediction and functional annotation, allowing accurate smBGC mining. To increase the value of current public databases for validation and reproducibility, revised strict guidelines for genome length, $\mathrm{G} / \mathrm{C}$ content, and gene space completeness are required.

\subsection{Identification of smBGCs encoded in Streptomyces genomes}

The development of nucleotide or amino acid sequence alignment tools (e.g. BLAST, diamond, and HMMer) significantly accelerated the smBGC mining process, resulting in the accumulation of smBGC information and the construction of several smBGC databases such as DECIPHER, ${ }^{21}$ DoBISCUIT, ${ }^{22}$ and MIBiG. ${ }^{23}$ Since then, several in silico smBGC mining tools have been developed, including BAGEL, ${ }^{24}$ ClusterScan, ${ }^{25}$ NP.searcher, ${ }^{26}$ CLUSEAN, ${ }^{27}$ PRISM, ${ }^{28}$ and antiSMASH. ${ }^{29}$ Some of them are specialised for seeking specific types of smBGCs. For example, BAGEL is for ribosomally synthesised and posttranslationally modified peptides (RiPPs) such as bacteriocins. ${ }^{24}$ The latest versions of antiSMASH and PRISM, which are webbased user-friendly in silico smBGC mining tools, can identify up to 52 and 22 different types of smBGCs, respectively. ${ }^{30,31} \mathrm{~A}$ limitation of these tools is that they rely on rule-based predictions, which are restricted to detect well-characterised smBGC classes. To overcome this limitation, machine learningbased prediction tools, such as ClusterFinder ${ }^{32}$ and DeepBGC ${ }^{33}$, have been developed, which have the potential to identify novel class smBGCs. However, two factors hampering the detection of completely unknown types of smBGCs are considerably high false-positive rates and known clusters of positive training sets. The detailed algorithms and history of smBGC mining tools have been comprehensively reviewed several times; ${ }^{34,35}$ this section will focus on elucidating the richness of unexplored smBGCs in currently available Streptomyces genomes using in silico genome mining tools.

As the number of available genomes increased to 1,926, we revisited the biosynthetic diversity of Streptomyces genomes using two in silico genome mining tools, antiSMASH and PRISM. A total of 70,253 and 51,976 smBGCs were predicted using antiSMASH version 5 and PRISM version 4 , respectively. In the case of the aforementioned $S$. cattleya 1093_SCAT (GCA_001070095.1) genome, neither tool detected any smBGCs, further supporting the hypothesis that this genome is unusable. The longer the genome length, the more smBGCs tended to be detected; however, genomes with abnormally
A

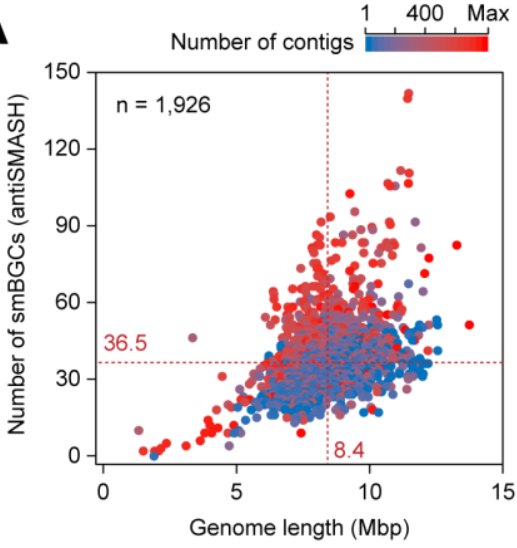

B

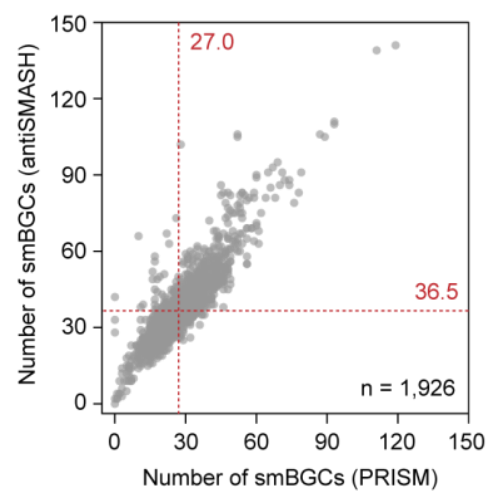

C

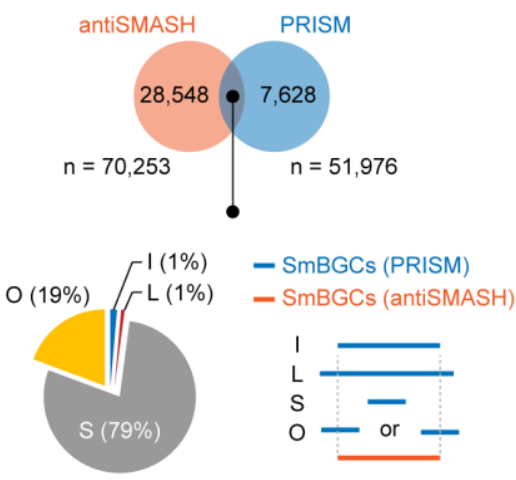

Fig. 4 Mining smBGCs from 1,926 genomes using antiSMASH and PRISM. (A) Influence of genome quality and length on smBGC prediction. (B) Comparison of predicted smBGC numbers by genome using two representative in silico genome mining tools, antiSMASH and PRISM. (C) Comparison of smBGCs predicted by antiSMASH and PRISM based on genomic position. I: identical; L: long; S: short; O: overlapped. 
small or large numbers of smBGCs often comprised numerous contigs, supporting the importance of genome quality for accurate smBGC prediction (Fig. 4A). The number of smBGCs per genome mined by antiSMASH (36.5 on average) and PRISM (27.0 on average) showed a high correlation, but antiSMASH detected more smBGCs than PRISM (Fig. 4B). Next, we compared smBGCs predicted from antiSMASH and PRISM analyses based on their genomic positions. A total of 41,705 antiSMASH smBGCs matched 44,348 PRISM smBGCs (Fig. 4C). AntiSMASH usually defines a wider genomic region of smBGC compared to PRISM; therefore, several smBGCs from PRISM are often included in a single smBGC from antiSMASH. The median value of smBGC length from antiSMASH was $21.1 \mathrm{kbp}$, which was longer than that of smBGCs from PRISM (9.6 kbp). In addition, $79 \%$ of matched smBGCs from PRISM were shorter than matched smBGCs from antiSMASH (Fig. 4C).

NRPS, PKS, NRPS-PKS hybrid, lanthipeptide, and butyrolactone-type smBGCs were dominant among the smBGCs predicted by antiSMASH and PRISM. These are the types of smBGCs that have been extensively studied, indicating that rich information is important for in silico genome mining. Meanwhile, most of the terpene, indole, and bacteriocin-type smBGCs were uniquely predicted by antiSMASH and rarely detected by PRISM, implying that the two tools have different detection capabilities depending on the types of smBGCs (Fig. 5A). Unlike PRISM, antiSMASH compares detected smBGCs with well-established smBGCs in the MIBiG database and provides the similarity value with the most similar smBGCs. Accordingly, 52,778 antiSMASH smBGCs were matched with the smBGCs in the current MIBiG database. The tRNA-derived peptide, lipopeptide, terpene, and RiPP encoding smBGCs showed high similarity with the matched smBGCs in the database, indicating that, in the case of these types, databases are sufficiently established or the algorithm is designed to detect only smBGCs highly similar to well-known smBGCs (Fig. 5B). The similarity of saccharide type smBGCs was mostly low, which means that they have been insufficiently characterised. Meanwhile, although a large number of NRPS- and PKS-type smBGCs were matched

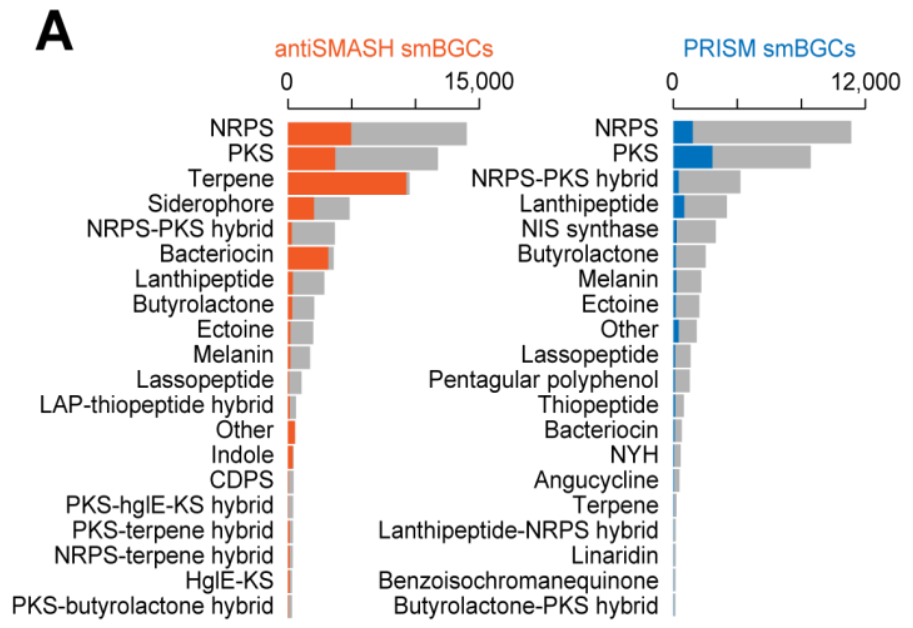

Predicted by both tools $\square$ Predicted by only antiSMASH — Predicted by only PRISM with the current database, the average similarity was as low as $30 \%$, suggesting that unexplored NRPs and PKs are abundant and that these smBGCs have great potential to produce novel SMs.

Ultimately, predicting the chemical structure of the products of smBGCs from genetic information relieves the burden on experimental validation approaches. In this regard, the representative smBGC mining tools, antiSMASH and PRISM, offer chemical structure prediction of smBGC products. Both tools connect putative substrates considering substrate specificity and order of catalytic domains, and estimate the modification governed by nearby tailoring enzymes. ${ }^{30,31}$ Structure prediction is available for dozens of smBGC types. However, accurate structure prediction is still often hampered by the variety of post-assembly modifications (e.g. methylation, cyclisation, and condensation). We believe that the accumulation of mechanistic studies on various smBGC types has allowed improving prediction algorithms continuously. Improvement of existing database will increase the accuracy of structure prediction and accelerate novel SM discovery from unexplored smBGCs in Streptomyces genomes.

\subsection{Characterisation of natural products synthesised by the smBGCs}

Advances in genome mining tools have enabled the rapid discovery of unexplored smBGCs from Streptomyces genomes, which have the potential to produce novel SMs. To make these discoveries worthwhile, characterising products of the smBGCs, called the forward approach (genes to metabolites), must be followed. One noteworthy example of the forward approach is the discovery of the tris-hydroxamate tetrapeptide iron chelator, coelichelin, from S. coelicolor (Fig. 6). ${ }^{36,37}$ A novel NRPS-type smBGC was discovered in the $S$. coelicolor genome by searching for NRPS-like genes. The chemical structure of the encoded product was roughly determined based on the prediction of adenylation domain substrate selectivity. Next, inactivation of the core biosynthetic gene, $c c h H$, followed by

B

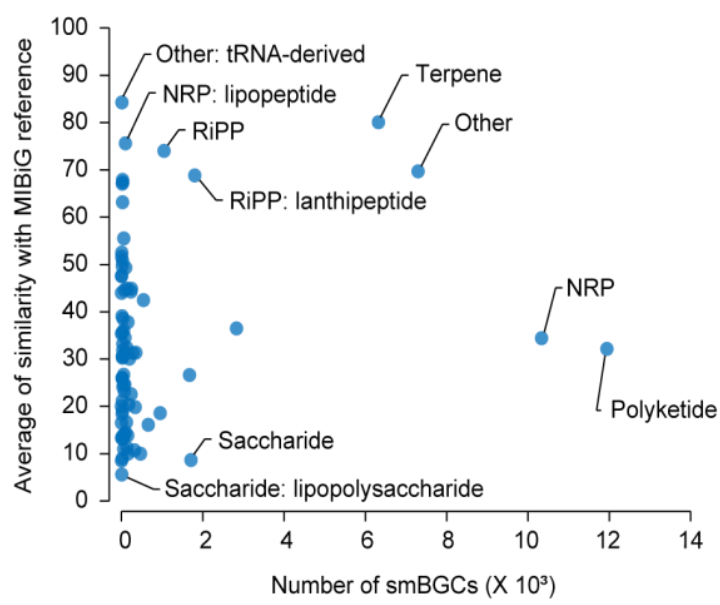
database. 
comparative metabolic profiling, tandem mass spectrometric analysis, and NMR analysis determined the exact chemical structure of coelichelin. Finally, heterologous expression of cchH in S. fungicidicus B-5477 resulted in the production of coelichelin. As in the case of coelichelin discovery, the strategy for connecting unexplored smBGCs to products generally exploits comparative metabolic profiling after altering the expression level of smBGC-encoded genes. For example,
PKs<smiles>CC(=O)NC(CS/C(C)=C/C=C1\C=Cc2ncccc21)C(=O)O</smiles>
S. argillaceus ATCC 12956<smiles>O=C(O)CC1Cc2cc3cccc(O)c3c(O)c2C(=O)O1</smiles>

Streptoketide S. sp. Tü 6314

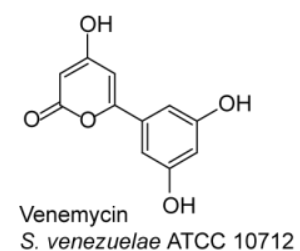<smiles>COc1cccc2cc3c4c(ccc3cc12)CC(C)(O)CC4=O</smiles>

S. sp. W007<smiles>CCc1cc(O)c(CC)c(=O)o1</smiles>

S.<smiles>C=CC(C)(C)C1=CC(O)Oc2c1cc(O)c1c2CCC(C)(C)O1</smiles><smiles>CC(=O)C(O)C(C)C(C)CCC=CCCc1cnco1</smiles>

Phthoxazolin A

S. avermitilis KA-320

\section{NRPs}<smiles>CC(=O)N(C(=O)C(CCCN(O)C=O)NC(=O)C(N)CCCN(O)C=O)C(CCCN(O)C=O)C(=O)NC(CCCNC(=N)N)C(=O)N(O)CCCCN</smiles><smiles>CC(O)C(NC(=O)C(N)CCCN(O)C=O)C(=O)N(O)CCCC(NC(=O)C(N)CCCN(O)C=O)C(=O)O</smiles>

\section{Lanthipeptides}

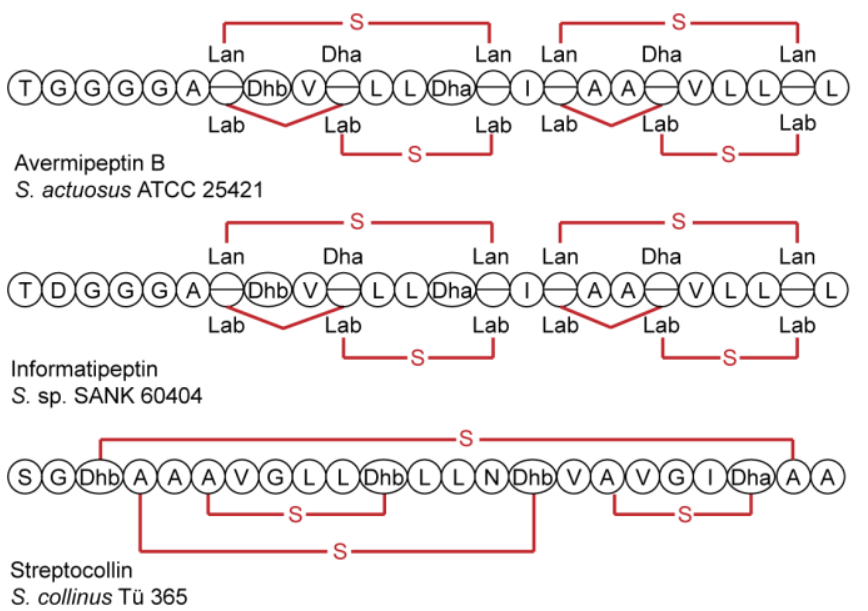

\section{Phenazines}<smiles>COc1ccc(OC(=O)c2cccc3nc4c(C(=O)O)cccc4nc23)c(O)c1</smiles>

Novel phenazine antibiotic S. kebangsaanensis

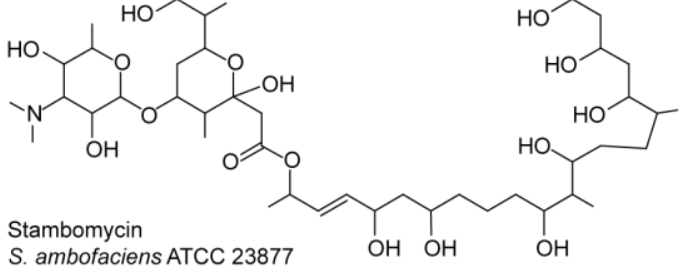

\section{Thiopeptides}

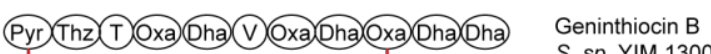 S. sp. YIM 130001

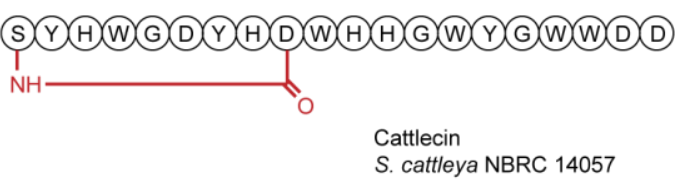

Fig. 6 Chemical structure of SMs discovered through genome mining and smBGC characterization. Dha: dehydroalanine; Dhb: dehydrobytyrine; Pyr: pyridine; Thz: thiazole; Oxa: oxazole; Lan: lanthionine; Lab: labionin. 
genome sequencing and mining of $S$. netropsis DSM40846 revealed two scattered novel smBGCs, suspected to be involved in pyrrolamide production. ${ }^{38}$ Functional analysis by deleting 13 smBGC-encoded genes revealed that the products of smBGCs are three pyrrolamides, including congocidine, distamycin, and disgocidine. Furthermore, the results of gene deletion experiments revealed genes involved in the assembly of pyrrolamides, precursor biosynthesis, and tailoring pyrrolamides, suggesting a biosynthetic pathway model for the three pyrrolamides (Fig. 6).

In most cases, smBGCs are silent and quantities of products are likely to be below the detection limit of most analytical tools. Since smBGCs commonly contain several genes, the most efficient way to activate the expression of entire smBGCs is to up-regulate the cluster-situated positive regulator or downregulate the negative regulator. ${ }^{39}$ For example, genome sequence analysis of $S$. ambofaciens ATCC 23877 revealed a type I PKS BGC comprising 25 genes, which are not expressed under laboratory culture conditions. Constitutive expression of cluster-situated regulators (CSRs) activated the transcription of entire smBGCs and resulted in the production of novel SMs, named stambomycins (Fig. 6). ${ }^{40}$ As another example, constitutive expression of LuxR-like family transcriptional activators encoded in cryptic BGCs of $S$. venezuelae resulted in the production of novel biaryl polyketides, named venemycin (Fig. 6). ${ }^{41}$ For smBGC activation, the recent application of the CRISPR/Cas system to the Streptomyces species has remarkably expedited the characterisation of novel smBGCs. ${ }^{42,43}$ CRISPR/Cas9 mediated promoter refactoring has been applied to several Streptomyces species to activate different classes of smBGCs, including PKS, NRPS, NRPS-PKS hybrid, and phosphonate clusters, uncovering unique metabolites. ${ }^{44}$ In addition, CRISPR based transcriptional repression and activation systems have the potential to link genotype to phenotype without conventional genetic manipulation. The transcriptional repression system (i.e. CRISPRi) uses endonuclease-deficient Cas9 (dCas9) to bind the target gene, and the transcriptional activation system (i.e. CRISPRa) uses dCas9 linked with transcriptional activators to recruit RNA polymerase to the target gene promoter region. CRISPRi has been applied to Streptomyces several times to regulate SM productions, including ACT, RED, calcium-dependent antibiotic (CDA), and yellow-pigmented cryptic polyketone (CPK) in S. coelicolor, ${ }^{45-47}$ and rapamycin in S. rapamycinicus. ${ }^{48}$ Meanwhile, CRISPRa has not been applied to Streptomyces. Bacterial CRISPRa is challenging due to strict target site requirements. ${ }^{49}$ Bacterial CRISPRa was only effective at target sites located in a narrow 40 base window between 60 and 100 bases upstream of the TSS, whereas eukaryotes have broad effective window between 400 and 50 bases upstream of the TSS. Moreover, even within the narrow 40 bases window, only a few precisely positioned target sites are effective with a periodicity of 10-11 bases corresponding to one helical turn of DNA. Additional factors, such as the strength of the target promoter, the sequence composition upstream of the TSS, and interruption of binding by other transcription factors at target sites also affect the CRISPRa activity. Just as the Streptomyces genome has complex transcription regulatory elements and networks, it may also have strict requirements for the target site. In the current situation, testing as many sites as possible by increasing the number of available target sites by applying dCas12a with alternative PAM specificities, ${ }^{46}$ or expanded PAM variants of dCas $9^{50}$ can be a solution.

If native hosts of discovered smBGCs are unculturable or genetic manipulation is unavailable, heterologous expression could be a suitable alternative. ${ }^{51}$ For heterologous expression of the target smBGCs, Streptomyces is generally used as a host because it has abundant precursor and cofactor pools for SMs, post-modification systems, broad antibiotic resistance, and similar levels of G/C content and codon usage. ${ }^{52,53}$ Importantly, it enables the expression of smBGCs that are not expressed in the native host by avoiding the innate regulatory network of the native host. ${ }^{53}$ Several genetically amenable Streptomyces species are widely used as heterologous expression hosts, such as S. coelicolor, S. lividans, S. avermitilis, S. albus, and S. venezuelae. ${ }^{54}$ However, the presence of large numbers of

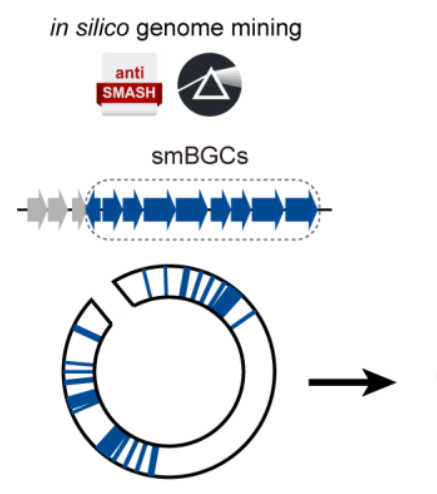

WT

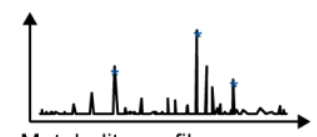

Metabolite profile

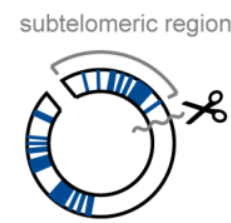

Large deletion

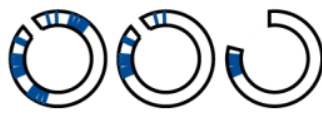

Serial deletion

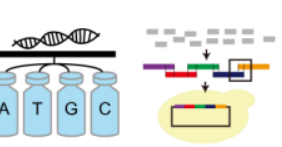

Genome synthesis

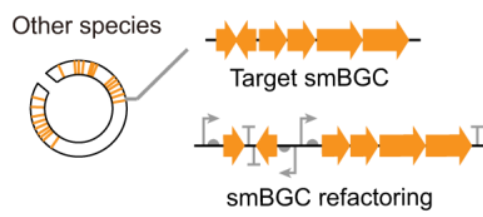

smBGC refactoring

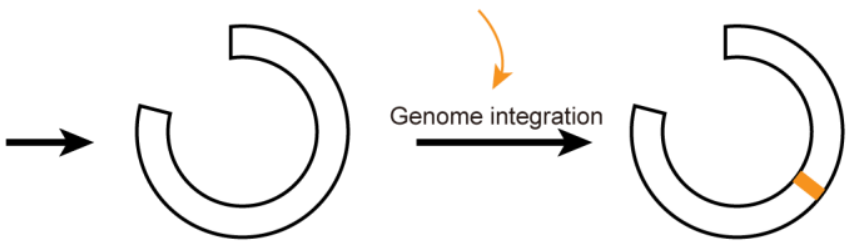

Genome minimized chassis strain

Heterologous expression

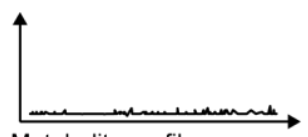

Metabolite profile

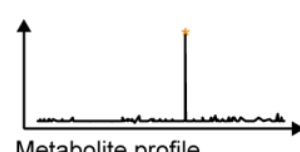

Metabolite profile

Fig. 7 Construction of rationally designed chassis strains. Based on the smBGC prediction using in silico genome mining tools, rational chassis strain design became available through genome engineering or genome synthesis. 
endogenous smBGCs may interfere with the high expression of the desired smBGCs by regulatory crosstalk or precursor competition between endogenous and heterologous smBGCs. To overcome this, Streptomyces hosts have been reconstructed as chassis strains for heterologous expression by removing nonessential genes (or genomic regions) and/or several endogenous smBGCs. The resulting genome-reduced chassis strains exhibit simple metabolite profiles, high intracellular ATP, and high cofactor levels, facilitating improved production of heterologous metabolites (Fig. 7). ${ }^{55-59}$

Accurate genome sequence and smBGC prediction have enabled the rational design of genome-reduced chassis strains. First, by comparing the whole genome sequences of Streptomyces species, subtelomeric regions located at the ends of the linear chromosome were predicted to be non-essential, and contain a large portion of endogenous smBGCs. ${ }^{60}$ Second, large numbers of endogenous smBGCs were predicted through in silico genome mining and serially deleted in the genome. The first reported genome-reduced Streptomyces chassis strains were S. avermitilis SUKA5, SUKA17, and SUKA22, which more than $1.4 \mathrm{Mbp}$ regions were serially removed from $S$. avermitilis chromosome, including left telomeric arm. ${ }^{55,61}$ Other historical genome-reduced Streptomyces chassis strains were S. coelicolor M1152 and M1154 superhost strains of which four smBGCs (ACT, RED, CDA and CPK) were deleted and additionally contained mutations at $r p o B$ and $r p s L$ genes in order to increase the yield of heterologous production. ${ }^{56}$ Indeed, these strains produced 20-40 times more chloramphenicol than the parental strain. Due to their ease of culturing and genetic manipulation, they have been broadly used for both improved production and characterization of SM biosynthetic pathways. ${ }^{62}$ More recently, 15 endogenous smBGCs were removed from the genome of $S$. albus J1074, producing S. albus Del14, which was successfully used to characterise the products of cryptic pyridinopyrone BGC from S. albus subsp. chlorinus NRRL B-24108 and two cryptic smBGCs of the Frankia strains. ${ }^{57}$ As another example, the $S$. lividans $\triangle Y A 9$ strain, of which nine endogenous smBGCs were deleted, produced new representatives of the pyrrolobenzodiazepines family by expressing the BAC library of the S. albus subsp. chlorinus NRRL B-24108 genome. ${ }^{59}$ Interestingly, when the same BAC library was expressed in $S$. albus Del14, the identified metabolites were different with $S$. lividans $\triangle$ YA9 strain, indicating that SM production capacity differs between heterologous expression hosts. ${ }^{59}$ The present bottleneck for the construction of the cluster-free chassis is the time-consuming and laborious genetic modification. For example, the construction of S. coelicolor ZM12, of which 10 endogenous smBGCs and $900 \mathrm{kbp}$ subtelomeric regions were deleted, spanned about 6 years. ${ }^{63}$ This limitation can be alleviated by applying the CRISPR/Cas system, which allows serial deletion without additional steps because it does not leave a scar like a resistance marker.

In addition, we can consider recent progress in DNA synthesis technology, enabling the construction of artificial synthetic genomes as a bottom-up approach. In the case of bacteria, the genome of Mycoplasma mycoides $(1,079 \mathrm{kbp})^{64}$ was chemically synthesised and a minimally designed version (531 kbp) ${ }^{65}$ was successfully constructed. Recently, the G/C-rich chromosome of Caulobacter ethensis (57\%) was redesigned and chemically synthesised (785 kbp) by rewriting 123,562 codons. ${ }^{66}$ We expect that in silico redesign and de novo writing of the Streptomyces genome will enable the rational construction of genome-minimised chassis strains. The DNA synthesis technology is also advantageous for heterologous
Tn-Seq
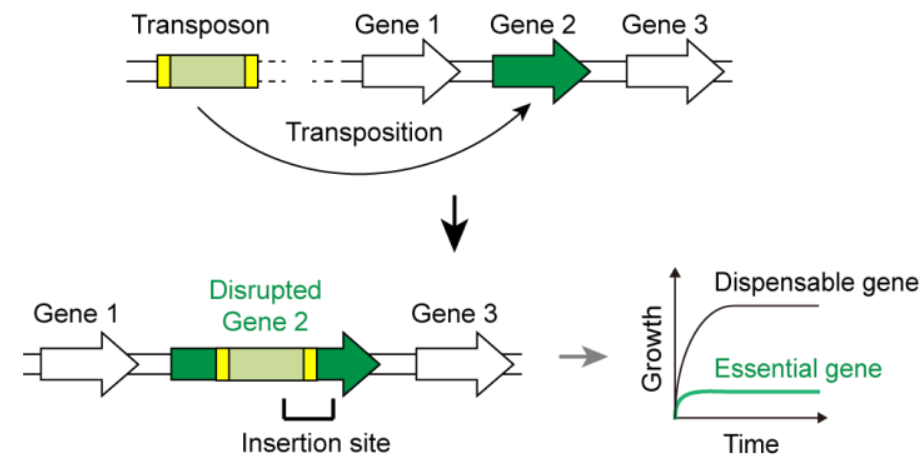

$\downarrow$

Insertion sites sequencing reads

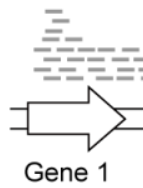

Fitness calculation

Dispensable

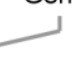

ene

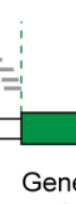

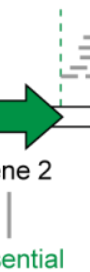

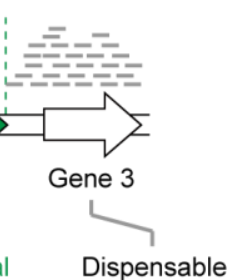

\section{Genome-wide CRISPRi}
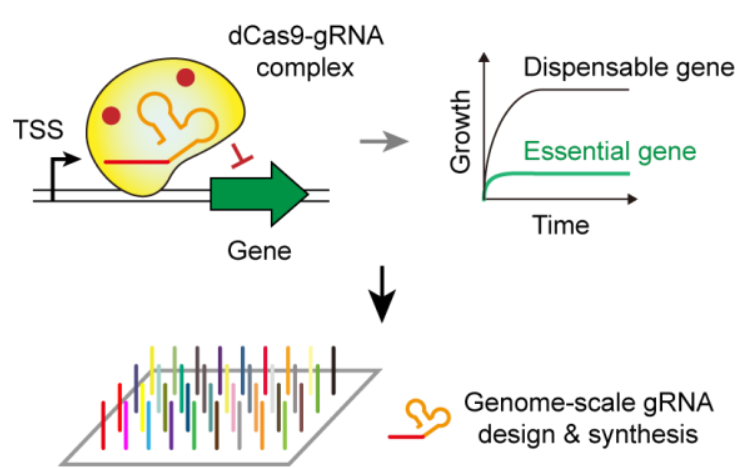

$\downarrow$

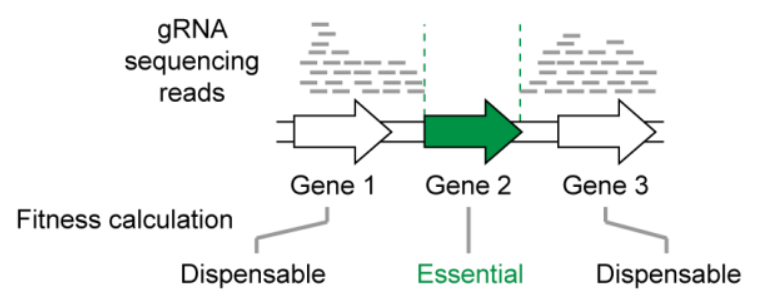

Fig. 8 Measurement of genome-scale gene fitness using Tn-Seq and CRISPRi. Fitness of each gene can be estimated by the degree of transposon insertion or proportion of gRNA cassette for CRISPRi. 
expression in terms of smBGC refactoring, including promoter exchange, which guarantees the considerable expression of smBGCs, supporting characterisation of the final products in heterologous hosts. ${ }^{67}$ However, synthesizing G/C-rich DNA is still challenging due to the stable single-stranded DNAs' secondary structures and thermal stability of double-stranded DNA due to the inter- and intra-base stacking, resulting resilience to denaturation. ${ }^{68}$ Even if gene synthesis is performed at a high temperature to deal with the high stability, mispriming and misassembling problems occur more often. So far, lowering the $\mathrm{G} / \mathrm{C}$ content of $\mathrm{smBGC}$ by codon rewriting or fine temperature control during the DNA synthesis process are possible strategies for $\mathrm{smBGC}$ synthesis. Considering that Streptomyces species are promising heterologous expression hosts as mentioned above, if the DNA synthesis technology is advanced to synthesise long length and high G/C content DNA, product characterisation through $\mathrm{smBGC}$ refactoring will be further accelerated (Fig. 7).

\subsection{Understanding gene function and fitness using functional genomics}

Streptomyces genomes contain abundant hypothetical genes whose functions are not experimentally validated. ${ }^{69}$ Therefore, to expand our understanding of secondary metabolism, a functional genomics approach is required to understand the functions of the unknown genes involved in secondary metabolism. With the recent advances in high-throughput sequencing technology, for example, comparative genomics approaches can be performed at nucleotide resolution, often leading to the identification of unexplored components for desired phenotypes, including high production of SMs.

Conventionally, strain engineering for SM production relies on repeated non-targeted random mutagenesis followed by screening high producers. However, the underlying genetic basis for the phenotypic variations were mostly hidden. ${ }^{70}$ The ease of assessing the complete genome sequence enabled the determination of the full genome sequence of the highproducing mutant, leading to the identification of the causal mutations for high production of the target SM and further engineering of the industrial mutant. For example, the genomes of two clavulanic acid (CA) high-producing S. clavuligerus strains, obtained from random mutagenesis, and their parental wildtype strains were sequenced and compared. ${ }^{71}$ Although the causal mutations for high production were not fully determined, a frameshift mutation was found in a CA biosynthetic gene, cas1, of the industrial high producer, and by introducing wild-type cas1, the production of CA by industrial high producers was further improved. In addition, competition-based adaptive laboratory evolution (ALE) of $S$. clavuligerus by co-culturing with pathogenic bacteria, methicillin-resistant Staphylococcus aureus N315, led S. clavuligerus to acquire the ability to produce the antibiotic holomycin. Whole genome re-sequencing and comparative genomics revealed that the loss of the large plasmid (pSCL4) and several single nucleotide polymorphisms in genes affected SM biosynthesis. ${ }^{72}$
As another functional genomics approach, high-throughput transposase-mediated mutagenesis systems, followed by screening transposon insertion sites on a genome-scale using NGS (i.e., Tn-Seq), enabled the determination of gene essentiality and the further exploration of SM productionrelated genes. Transposon-related studies in Streptomyces species have been primarily conducted in S. coelicolor. ${ }^{73,74}$ For example, using a transposase-based Tn5 transposition system, a $S$. coelicolor mutagenesis library covering 51,443 transposon insertions was constructed. About 724 mutants including transposon insertions in 365 genes altered the level of production of undecylprodigiosin (RED), indicating that hundreds of unexplored genes affected the RED production of S. coelicolor. ${ }^{75}$ However, resolution of the data was low and insufficient to distinguish essential gene sets of $S$. coelicolor. In addition, transposase-mediated mutagenesis often showed a preference for locations with a relatively low $\mathrm{G} / \mathrm{C}$ content in core chromosome regions. ${ }^{75} \mathrm{~A}$ recently developed genomescale CRISPRi platform could overcome this limitation (Fig. 8). ${ }^{45,52,76}$ Taken together, the genomics of Streptomyces is not only essential for mining smBGCs but is also required to expand knowledge about secondary metabolism and increase the production of target SMs. Moreover, genomics can be a starting point for further systematic approaches that will be described below.

\section{Transcriptomics: Elucidation of transcriptional regulation on secondary metabolism}

The secondary metabolism of Streptomyces is subject to complex transcriptional regulation, and many smBGCs are silent under laboratory culture conditions. ${ }^{77}$ Thus, the transcriptional information of unexplored smBGCs is required for establishing future engineering strategies. For example, if an smBGC is transcriptionally inactive, it should be stimulated by changing conditions or refactoring transcriptional elements of the smBGC. ${ }^{44}$ Furthermore, secondary metabolism is tightly connected with primary metabolism and during cell growth, a dynamic metabolic switch from primary to secondary metabolism is observed. ${ }^{78,79}$ In this regard, assessing the transcriptome information is crucial for understanding the regulation of both primary and secondary metabolism. Currently, RNA sequencing (RNA-Seq) techniques are well established for various specimens, ranging from unicellular bacteria to multicellular eukaryotes. ${ }^{80,81}$ Compared to microarrays, RNA-Seq is quantifiable with high-resolution (nucleotide resolution) and independent of prior knowledge of gene sequences. Approximately 60 RNA-Seq analyses of Streptomyces have been reported, which can be categorised into (i) time-course transcriptome profiling according to growth and (ii) comparative transcriptomics for different conditions. In this section, we describe representative examples of transcriptome analysis of Streptomyces using RNA-Seq and highlight future perspectives. 


\subsection{Time-course transcriptome profiling and genetic development}

The metabolic switch of Streptomyces from primary to secondary metabolism occurs with the growth phase transition from exponential to stationary phase. During exponential growth, Streptomyces produce metabolites required for cell maintenance. When essential nutrients, such as carbon, nitrogen, and phosphate are limited, Streptomyces stops growing and rearranges its entire metabolism to produce SMs using primary metabolites as building blocks. For example, $S$. coelicolor decreases the expression of genes related to protein biosynthesis and nitrogen metabolism during the metabolic switch, whereas the expression of genes encoding actinorhodin (ACT) and undecylprodigiosin (RED) BGCs are up-regulated. ${ }^{82,83}$ The metabolic switch of Streptomyces is also associated with differentiation; for instance, the expression of development (e.g. sporulation) related genes increased during the early growth phase and started to decrease before the metabolic switch. ${ }^{82}$ Although the relationship between differentiation and secondary metabolism is poorly understood, in the case of ACT and RED, differentiation is known to activate production. ${ }^{84}$ Overall, understanding this complex cellular process during growth is required to understand and optimise the production of valuable SMs.

Reorganising metabolism during growth transition is achieved by altering large sets of gene expression. To profile the change in transcriptional dynamics according to growth, expression levels of the individual genes have been measured using RNA-Seq. ${ }^{69,78,85,86}$ During the metabolic switch, downregulation of translation machinery genes and up-regulation of genes related to stress response, especially nutrient depletion, were consistent between time-course transcriptomic profiling studies. ${ }^{69,85}$ Most Streptomyces species, including $S$. coelicolor, ${ }^{78}$ S. lividans, ${ }^{86}$ S. clavuligerus, ${ }^{69}$ S. xiamenensis,,${ }^{87}$ and S. fungicidicus ${ }^{88}$ are continually up-regulated in the expression of smBGC-encoded genes after exponential growth. However, not all smBGCs were activated during the metabolic switch. In particular, transcriptome profiling of S. albus J1074 at three different time points revealed that among the 22 smBGCs of $S$. albus J1074, only the ectoine BGC was activated during the metabolic switch and the remaining smBGCs were silent. ${ }^{85}$ These results emphasise that each smBGC is subjected to complex and distinct regulations, and transcriptional profiling is necessary to determine whether smBGCs of interest are being expressed under certain experimental conditions. Additionally, time-course transcriptional profiling allows us to define ratelimiting genes for SM production. For example, RNA-Seq analysis of S. fungicidicus TXX3120 at four different time points during fermentation revealed that genes involved in enduracidin biosynthesis were increased according to growth, except for a core biosynthetic gene, endC, which showed remarkably lower expression levels than others. ${ }^{88}$ Indeed, subsequent overexpression of endC enhanced enduracidin production by more than two-fold compared to the parental strain
Another use of time-course transcriptome data is for the rational development of genetic parts, especially promoters. RNA-Seq measures the transcriptional levels of all genes that are primarily determined by the promoter sequence of each gene; therefore, time-course RNA-Seq data allows for the screening of strongly and constantly expressed genes at multiple time points, of which promoters are reasonable candidates for constitutive promoters. To date, only a few strong constitutive promoters, commonly ermEp*, kasOp* and gapdhp, have been utilised for gene expression in Streptomyces, but their expression strength and consistency has been questioned repeatedly. ${ }^{89}$ Therefore, the identification of various native constitutive promoters is required for the reliable genetic engineering of Streptomyces. In the case of $S$. albus J1074, 32 candidates for strong promoters were discovered based on RNA-Seq data under two culturing conditions and two time points. A catechol 2,3-dioxygenase (XYIE)-based examination of promoter strength revealed that 10 out of 32 promoters have stronger expression levels than ermEp* and four promoters showed constitutive activity..$^{90}$ Additionally, RNA-Seq-based rational selection of putative constitutive promoters from S. coelicolor M145, resulted in the discovery of 166 putative constitutive promoters, which are likely to be constantly expressed in other Streptomyces, including $S$. venezuelae WVR2006 and S. albus J1074. ${ }^{89}$

However, expressing smBGCs under strong constitutive promoters overlooks the metabolic switch of Streptomyces during growth. When smBGCs are strongly activated before the accumulation of building blocks from primary metabolism, efficient SM production is sometimes restricted and even toxic for cell growth. ${ }^{91,92}$ In this regard, the development of temporal promoters with various strengths is desirable for optimal SM production in Streptomyces. As far as we know, time-course RNA-Seq data-based temporal promoter development has not been reported, but there is a case based on microarray data. In this study, the overexpression of the actll-orf4 gene (the specific activator of ACT BGC in S. coelicolor) under nine constitutive promoters proved that ACT production level was weakly correlated with the strength of promoters. ${ }^{93}$ Consistent results were observed in the same experiment on jadJ, the jadomycin $\mathrm{BGC}$ activator of $S$. venezuelae. Expressing the two activators under the inducible promoters while altering the induction time and strength revealed that the induction time and strength are critical for optimal production of both ACT and jadomycin. In fact, inducible promoters are not suitable for industrial use because inducer treatment increases production costs. Therefore, through time-course transcriptome analysis, 24 native promoters similar to the optimal induction condition with respect to strength and expression initiating time were selected. Among the promoters, 15 resulted in higher ACT production compared to an inducible promoter under optimal conditions. 93

Taken together, time-course RNA-Seq data have been utilised to understand the metabolic switch of Streptomyces during growth and to develop constitutive promoters with various strengths. Furthermore, the development of temporal promoters applicable to various Streptomyces species based on 
time-course RNA-Seq data will be very helpful in increasing the SM production capability of Streptomyces.

\subsection{Comparative transcriptomics for understanding regulation of secondary metabolism}

Complex and interlacing regulations on secondary metabolism are mostly triggered by various biotic (microbial, physical, or chemical interactions) or abiotic (temperature, $\mathrm{pH}$, pressure or nutrient depletion) environmental stresses. In laboratory culture conditions, Streptomyces keeps its secondary metabolism silent with a lack of those stimuli. A common approach for understanding the regulation of secondary metabolism is to compare the global transcriptome after (i) altering culture conditions or (ii) changing the expression levels of transcriptional regulators that are thought to be related to secondary metabolism. In this respect, approximately 40 RNASeq based comparative transcriptomic analyses have been conducted in Streptomyces from 2011 to 2020.94-129

First, modifying culture conditions, such as nutrient source change, elicitor treatment, $\mathrm{pH}$ shock, or co-culture with other organisms, is the simplest way to awaken or increase SM production. Comparative transcriptomic approaches allow the identification of a set of genes whose expression is influenced under specific conditions, providing insight into the underlying mechanisms. Elucidating the underlying mechanisms between culture conditions and SM production provides valuable information about regulation and unknown genes related to secondary metabolism.

Elicitors are defined as chemical compounds, which induce the biosynthesis of various SMs and actually, SMs themselves are often used as an elicitor. Specifically, antibiotics alter the transcription level of many genes when treated below the subinhibitory concentrations, called hormesis. ${ }^{130}$ In Streptomyces, antibiotics act as auto-regulators of their own biosynthesis, cross-regulators of the biosynthesis of other SMs, and chemical elicitors. Thus, hormesis of antibiotics is an important part of the transcriptional regulatory network. ${ }^{131}$ For example, exposure to lincomycin enhanced the ACT production of $S$. coelicolor A3(2). Comparative transcriptomics revealed that lincomycin treatment up-regulated the expression of efflux pump encoding genes, boosting the intrinsic resistance mechanisms. Meanwhile, the expression level of housekeeping genes encoding ATP synthase and ribosomal proteins was down-regulated, increasing the intracellular NTP level that may affect global gene expression. ${ }^{132}$

As an example of $\mathrm{pH}$ shock, $\varepsilon$-poly-L-lysine $(\varepsilon-\mathrm{PL})$ produced by several Streptomyces, including S. albulus and S. griseofuscus, is used as a natural food preservative owing to its broad antimicrobial function against various gram-positive and gramnegative bacteria. ${ }^{133}$ During the fermentation of $S$. albulus, if the $\mathrm{pH}$ is maintained above $5, \varepsilon$-PL is not produced, indicating that $\mathrm{pH}$ is an important parameter for $\varepsilon$-PL production. ${ }^{134}$ In fact, when the $\mathrm{pH}$ is lowered below 4 (i.e. $\mathrm{pH}$ shock), the production of $\varepsilon$-PL increases dramatically, but the underlying mechanism remains ambiguous. ${ }^{104}$ Comparative transcriptomic analysis revealed that $\mathrm{pH}$ shock influences the expression of a wide range of genes related to unsaturated fatty acid synthesis, respiratory chain, and signal transduction systems. This dramatic change in gene expression ultimately increased membrane permeability, provided sufficient energy for $\varepsilon-P L$ production, and upregulated the expression of $\varepsilon$-PL producing genes ( $\varepsilon$-PL synthetase) through signal transduction.

As another example, co-culture of Streptomyces with other microbes co-residing in environmental conditions is a promising approach to activate silent smBGCs by offering complex and unpredictable stresses to Streptomyces via physical interaction or nutrient competition. Although much effort has been made to change the SM production of Streptomyces using a co-culture system, only a few studies have revealed the underlying mechanisms. ${ }^{135-137}$ Recently, comparative transcriptomic analysis on co-cultures of $S$. coelicolor and Myxococcus xanthus revealed that iron competition between the two microbes is a key trigger for the production of SMs by Streptomyces and further revealed that a novel gene distal from the ACT BGC, SCO6666, is involved in ACT production under co-culture conditions. Based on this information, the iron-restricted condition was applied to other Streptomyces species, and consequently, 21 cryptic smBGCs were activated. ${ }^{109}$

Second, gene transcription is regulated by various transcriptional regulators, including transcription factors and sigma factors, which bind to promoter regions. Therefore, suppressing or promoting the expression of transcriptional regulators influences the expression of genes. In this regard, comparative transcriptomic analysis after changing the expression of transcriptional regulators enables the determination of the set of genes regulated by the transcriptional regulator. Through this approach, a regulon of 12 transcriptional regulators of Streptomyces has been elucidated to date, including MtrA, ${ }^{122} \mathrm{PhoU},{ }^{125} \mathrm{LmbU},{ }^{129}$ and Crp. ${ }^{128}$ For instance, LmbU is a transcriptional regulator encoded in the lincomycin BGC of $S$. lincolnensis known as a pathway-specific regulator, that positively controls lincomycin production. Indeed, the ImbU deletion strain completely lost its lincomycin production ability. However, comparative transcriptomic analysis between the original strain and the $\mathrm{ImbU}$ deletion strain revealed that $\mathrm{LmbU}$ is a pleiotropic transcriptional regulator. $\mathrm{LmbU}$ not only positively regulates the expression of all genes in the lincomycin BGC but also controls other genes related to lincomycin biosynthesis, such as precursor synthesis, transportation, and redox metabolism. ${ }^{129}$ As can be seen from these findings, transcriptional regulators encoded in smBGCs are not always smBGC-specific, but have the potential to regulate various genes related to SM production. Therefore, revealing the regulons of these transcriptional regulators is important to understand intertwined cellular processes and to discover novel genes related to SM production.

\subsection{Determination of the regulatory elements encoded in Streptomyces genomes}

Transcription is controlled in the unit of transcripts, which means that not only a single gene but often clusters of genes 
are under the control of a single promoter. In particular, smBGCs of Streptomyces mostly comprise operons encoding a set of genes $(>5 \mathrm{~kb})$, which are generally transcribed into one polycistronic mRNA. Therefore, to understand genetic regulation in greater detail, it is necessary to identify the operon structure of genes. Determining genome-scale transcription units (TU) requires both $5^{\prime}$ - and 3 '-end information of all transcripts, which can be obtained by NGS-based (i) transcription start site (TSS) sequencing (dRNA-Seq) $)^{138}$ and (ii) 3 '-end sequencing (Term-Seq) methods, ${ }^{139}$ respectively.

dRNA-Seq has been applied to several Streptomyces species, including. S. coelicolor, ${ }^{78}$ S. venezuelae, ${ }^{140}$ S. tsukubaensis, ${ }^{141} \mathrm{~S}$. clavuligerus, ${ }^{69}$ and S. lividans. ${ }^{86}$ For example, dRNA-Seq analysis of S. coelicolor, S. clavuligerus, and S. lividans determined 3,570, 2,659 , and 1,978 TSSs in each genome, respectively. ${ }^{69,78,86}$ This genome-scale TSS information enables the determination of key genetic regulatory elements in nucleotide resolution, such as promoters and $5^{\prime}$ untranslated regions ( $5^{\prime}$ UTR), as well as novel transcripts such as small RNAs (sRNAs). ${ }^{142,143}$ Genome-wide promoter architecture, including promoter elements (e.g. -10

A

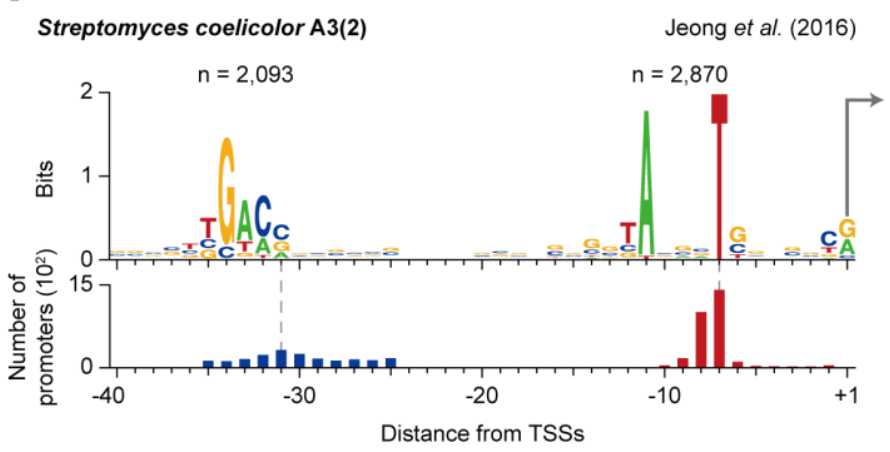

Streptomyces lividans TK24

Lee et al. (2019)

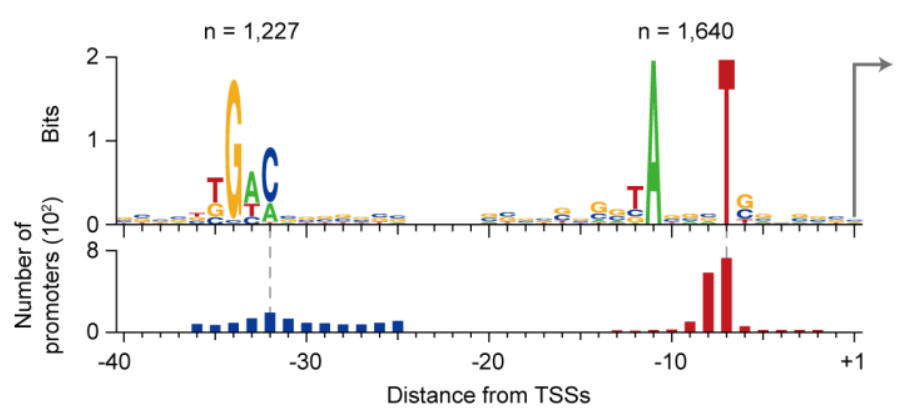

Streptomyces clavuligerus ATCC27064

Hwang et al. (2019)

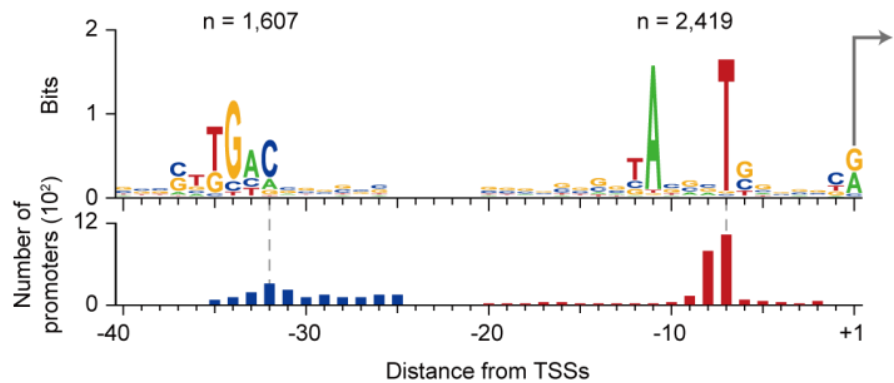

and -35 elements), is conserved across the Streptomyces species (Fig. 9A). ${ }^{69,78,86}$ The sequence of the -10 element was well-conserved in most promoters, and its motif was TANNNT. On the other hand, the -35 element sequence was not well conserved between promoters. Considering that the -10 and -35 elements are regions that the sigma factor recognises, and Streptomyces species have a large number of sigma factors (about 60 per species) compared to other bacteria, ${ }^{144}$ the less conserved -35 element sequence and the variable spacer length (i.e. distance between -10 and -35 elements) are important for determining the binding specificity of sigma factors to the promoters. Meanwhile, the region between the TSS and start codon, called the $5^{\prime}$ UTR, contains a ribosome binding site (RBS), which determines the translational efficiency of the downstream gene. The conserved RBS sequence of $S$. lividans was RRGGAG, ${ }^{86}$ which was almost identical to that of $S$. coelicolor ${ }^{78}$ and S. clavuligerus. ${ }^{69} \mathrm{~A}$ unique characteristic of the Streptomyces $5^{\prime}$ UTR is a considerable proportion of leaderless genes $\left(21 \%\right.$ in case of $S$. coelicolor) whose $5^{\prime}$ UTR length is shorter than $9 \mathrm{bp}$, which means that the $5^{\prime}$ ends of transcripts

B

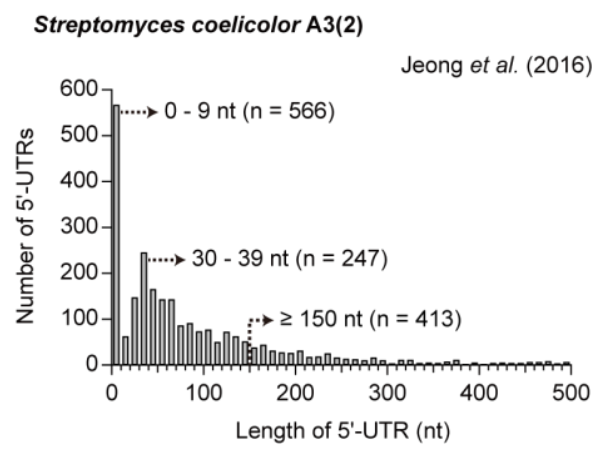

Streptomyces lividans TK24

Lee et al. (2019)

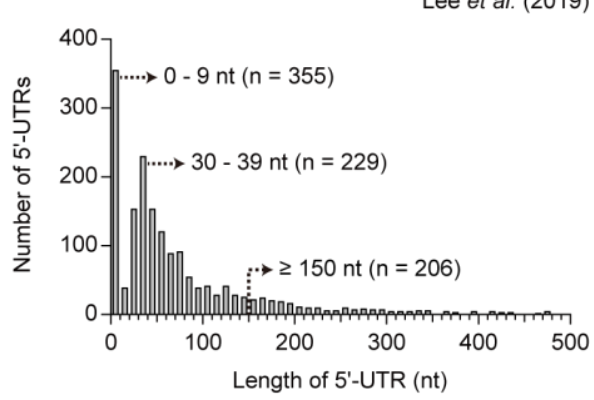

Streptomyces clavuligerus ATCC27064

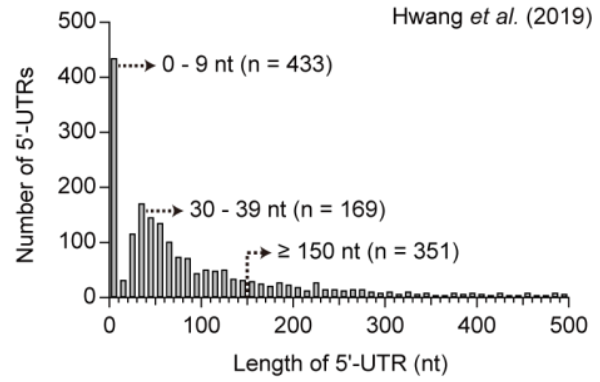

Fig. 9 Conserved regulatory elements of Streptomyces. (A) Conserved sequence of Streptomyces promoter elements ( -10 and -35$)$ and distance from TSSs revealed by dRNA-Seq result. (B) 5' UTR length distribution of three Streptomyces species. 
almost immediately start with the start codon (Fig. 9B). $69,78,86$ Many antibiotics inhibit the translation initiation step; therefore, it is suspected that Streptomyces, an active antibiotic producer, has a large number of leaderless genes providing resistance to these antibiotics.

In addition, TSS information is quite useful in developing genetic parts of Streptomyces. Examples of the development of the Streptomyces genetic parts described in section 3.1 did not consider TSS information; therefore, the upstream regions from the start codon were defined as promoters with a specific length, which actually contain both the promoter and $5^{\prime}$ UTR. The TSS information accurately distinguishes the transcriptional strength determined by promoters and the translational strength determined by $5^{\prime}$ UTR sequences, allowing us to develop the promoter and 5' UTR as independent genetic parts for synthetic biology applications. Indeed, in S. coelicolor, for which TSS information is available, the promoter and $5^{\prime}$ UTR were distinguished based on the TSS and the strength of each component was estimated based on transcriptome and translatome data. Finally, eight combinations with different transcriptional and translational strengths were constructed by combining two promoters and four $5^{\prime}$ UTRs. ${ }^{145}$

Until recently, TUs have been determined using only $5^{\prime}$ end information of the transcript. For example, in the case of the immunosuppressive drug FK506 producing S. tsukubaensis, nine TUs in the FK506 BGC were identified using TSS information. ${ }^{141}$ However, to determine the precise TUs, it is necessary to obtain the transcript $3^{\prime}$ end position (TEP) information and link it with the $5^{\prime}$ end information. Genome-scale TEP information can be secured using a recently developed RNA $3^{\prime}$ end sequencing method called Term-Seq. ${ }^{139}$ As it is a state-of-the-art method, it has only been applied to a few bacteria, ${ }^{146}$ and in the case of Streptomyces, it has only been applied to S. lividans. ${ }^{86} \mathrm{~A}$ total of 1,640 TEPs were identified in the $S$. lividans genome, revealing a sequence motif that contains a GC-rich region followed by a U-rich region, which resembles the Rho-independent transcription terminator of bacteria. ${ }^{147}$ The TEP information was then integrated with TSS information to define a total of 1,300 TUs, including 375 polycistronic TUs.

To date, only a few transcriptional terminators have been used as genetic parts in Streptomyces. ${ }^{148}$ The transcriptional terminator is the crucial genetic component for preventing transcriptional read-through to downstream genes. In particular, when the smBGC of Streptomyces comprises multiple operon structures, reliable transcription termination between TUs is required. The TEP information of Streptomyces obtained through Term-Seq will likely be used in the development of the native transcriptional terminator of Streptomyces as a genetic part in the future. The determination of the TU architecture by integrating TSS and TEP information enables us to reveal the presence of novel potential regulatory elements. The identified regulatory elements of individual TUs will broaden our understanding of complex regulatory mechanisms, allowing us to fully exploit the potential of Streptomyces.

\section{Interactomics: Construction of transcriptional regulatory networks}

As revealed by genomic and transcriptomic studies, Streptomyces possesses complex transcriptional regulatory systems that govern various biological processes, such as dynamic morphological development and metabolic shift from primary to secondary metabolism in response to environmental stimuli. These transcriptional regulatory systems comprise a large number of transcriptional regulators, including sigma factors and transcription factors, mainly responsible for transcriptional initiation. ${ }^{149,150}$ Compared to the small number of sigma factors in other bacteria, the Streptomyces genome usually encodes more than 60 sigma factors, including a great number of extra-cytoplasmic function sigma factors. ${ }^{150}$ Moreover, transcription factors are diverse in Streptomyces. ${ }^{150}$ Over several decades, genetic studies of Streptomyces mutants have defined the functions of pleiotropic regulators such as bld and whi genes whose mutants are defective in aerial mycelium formation and mature grey spore formation, respectively. ${ }^{151,152}$ In addition, CSRs of each SmBGC specifically regulate the expression of smBGC-encoded genes, for example, ActII-ORF4 for ACT biosynthesis. ${ }^{153}$ These numerous sigma factors and transcription factors are known to be hierarchically connected by autoregulation or cross-regulation of each other to construct a complex transcriptional regulatory network. ${ }^{153}$

Although a set of genes controlled by the transcriptional regulator can be obtained through comparative transcriptome analysis, to determine the detailed transcriptional regulatory network, identification of the direct binding targets for each transcriptional regulator is indispensable. In recent years, the 'regulon' of the transcriptional regulator, which is a group of genes regulated by the transcriptional regulator, was identified by ChIP-based approaches. ${ }^{154}$ Among these approaches, ChIPseq is a more unbiased, whole genome-covering technique based on NGS. In the last decade, 24 proteins have been selected as the ChIP-seq targets in Streptomyces, and most of them were performed in the model strain S. venezuelae ATCC 10712 and S. coelicolor A3(2) (Table 1). There are four sigma factors and 20 transcription factors, which can be subdivided into pleiotropic, secondary metabolism, developmental, and others, based on the functions of their regulon members.

\subsection{Sigma factors}

ChIP-seq studies for four sigma factors have been reported in Streptomyces, which are HrdB and SigE of S. coelicolor A3(2), WhiG of S. venezuelae ATCC 10712 and AntA of S. albus S4. HrdB is a principal sigma factor of Streptomyces which is orthologous to RpoD of E. coli. ${ }^{155}$ The ChIP-seq experiment of HrdB identified about 2,100 target genes out of a total of 8,152 genes, and subsequent kinetic modeling of $\mathrm{HrdB}$ binding to DNA confirmed that approximately 1,700 genes were under direct control. ${ }^{156}$ The conserved binding motif of $\mathrm{HrdB}$ was analysed as 5'KSRMS $(N)_{18}$ TANNNT- $3^{\prime}$, with significant enrichment for the -10 region. Functional analysis of the HrdB regulon revealed that its pleiotropic function was related to primary metabolism and vegetative growth, including numerous genes involved in 
morphological development and secondary metabolism, particularly polyketide biosynthesis. The ECF sigma factor SigE directly regulates about 200 target genes with distinct binding motifs (5'-AACC $\left.(\mathrm{N})_{16-17} \mathrm{CSTCY}-3^{\prime}\right)$. The majority of the identified genes encode proteins implicated in cell envelope and cell wall development regulation in response to stresses. WhiG of $S$. venezuelae ATCC 10712 revealed that there were only three target genes, whiH, whil and vnz15005, which repress late sporulation regulators. ${ }^{157}$ Also, ECF sigma factor AntA is the CSR of the antimycin SmBGC, whose ChIP-seq data revealed only one regulatory target across the whole genome located in the promoter region of antGF and antHIJKLMNO. ${ }^{158}$ Interestingly, WhiG binding in the S. chattanoogensis L10 genome was distinct from that in S. venezuelae ATCC 10712 and directly activated the transcription of two natamycin biosynthetic genes. ${ }^{159}$ This suggests that the homologous gene may have a completely different regulon, emphasising that independent ChIP-seq experiments are required for different species.

Although >60 sigma factors have been annotated in each Streptomyces genome, only four ChIP-seq studies have been reported. The small protein size of sigma factors and structural similarity between sigma factors may be hurdles for DNA enrichment by specific antibodies, resulting in abundant ChIPseq signal noise. To solve this problem, several protein tags such as FLAG- and myc-tags have been fused to the target protein to use an anti-tag antibody for the ChIP protocol. In addition, it should be considered that a large number of sigma factors are known to have cognate anti-sigma factors for controlling the DNA-binding activity of the sigma factor depending on the cellular status. ${ }^{157,158}$ Moreover, cross-talk between sigma factors should be further considered: multiple sigma factors may bind to the same target and a sigma factor may bind to the promoter of other sigma factors, representing their hierarchical regulatory network structure. ${ }^{160}$

\subsection{Transcription factors}

Of the twenty ChIP-seq studies for the transcription factors of Streptomyces, eight were pleiotropic regulators related to both development and secondary metabolism, four were related to secondary metabolism, and eight were related to development or other functions.

More than 500 genes were determined as the direct regulatory targets controlled by the $\gamma$-butyrolactone responsive pleiotropic regulator AdpA. These included not only the genes governing morphological differentiation but also the genes governing secondary metabolism of streptomycin, lantibiotics and terpenes. Two interesting examples of pleiotropic

Table 1 ChIP-seq studies in Streptomyces.

\begin{tabular}{|c|c|c|c|c|c|}
\hline Cat & Sub cat & Protein & Strain & Motif (5' to $\left.3^{\prime}\right)$ & Ref \\
\hline $\mathrm{SF}^{\mathrm{a}}$ & $\mathrm{D}, \mathrm{S}^{\mathrm{c}}$ & $\mathrm{HrdB}$ & S. coelicolor A3(2) & KSRMS(N) ${ }_{18}$ TANNNT & 156 \\
\hline SF & $\mathrm{D}^{\mathrm{d}}$ & SigE & S. coelicolor A3(2) & $\operatorname{AACC}(\mathbf{N})_{16-17} \mathrm{CSTCY}$ & 161 \\
\hline SF & $\mathrm{D}$ & WhiG & S. venezuelae ATCC 10712 & TAAA $(\mathrm{N}){ }_{15} \mathrm{GCCGATAA}$ & 157 \\
\hline SF & $\mathrm{Se}^{\mathrm{s}}$ & AntA & S. albus S4 & NA & 158 \\
\hline $\mathrm{TF}^{\mathrm{b}}$ & $\mathrm{D}, \mathrm{S}$ & AdpA & S. griseus IFO13350 & TGGCSNGWWY & 162 \\
\hline TF & $\mathrm{D}, \mathrm{S}$ & BldM & S. venezuelae ATCC 10712 & TCACcCgnncGgGTGA & 163,164 \\
\hline TF & $D, S$ & Whil & S. venezuelae ATCC 10712 & TGnnCCGnnCGGGTGA & 163 \\
\hline TF & $D, S$ & MtrA & $\begin{array}{l}\text { S. coelicolor A3(2) } \\
\text { S. venezuelae NRRLB-65442 }\end{array}$ & NA & 165,166 \\
\hline TF & $\mathrm{D}, \mathrm{S}$ & ScbR & S. coelicolor A3(2) & MSGYTTSTTD & 167 \\
\hline TF & $\mathrm{D}, \mathrm{S}$ & ScbR2 & S. coelicolor A3(2) & DYTYSTYSWS & 167 \\
\hline TF & $\mathrm{D}, \mathrm{S}$ & MacR & S. coelicolor A3(2) & TGAGTACnnGTACTCA & 168 \\
\hline TF & $\mathrm{D}, \mathrm{S}$ & WhiH & S. venezuelae ATCC 10712 & NA & 164 \\
\hline TF & $\mathrm{S}$ & FscRI & S. albus S4 & YTAGGKWWWHVMVNRR & 169 \\
\hline TF & $S$ & CagR & S. clavuligerus F613-1 & CGCNGCCG & 170 \\
\hline TF & $S$ & Lsr2 & S. venezuelae ATCC 10712 & No consensus, AT-rich & 171 \\
\hline TF & $\mathrm{S}$ & RsrR & S. venezuelae NRRLB- 65442 & $\begin{array}{l}\text { GAWNYSRSMSWYSNNYNNYCYSNKTW, } \\
\text { GWWCNCGGAC }\end{array}$ & 140 \\
\hline TF & $\mathrm{D}$ & BldC & S. venezuelae NRRLB-65442 & $\operatorname{AATT}\left(N_{3-4}\right)(C / G)$ & 172 \\
\hline TF & $\mathrm{D}$ & WhiA & S. venezuelae ATCC 10712 & GACAC & 173,174 \\
\hline TF & $\mathrm{D}$ & WhiB & S. venezuelae ATCC 10712 & GACAC & 174 \\
\hline TF & $\mathrm{D}$ & BldD & S. venezuelae ATCC 10712 & TNAC(N) $)_{5}$ GTNA & 175,176 \\
\hline TF & $\mathrm{D}$ & BldO & S. venezuelae ATCC 10712 & TGXAATTXCA & 177 \\
\hline TF & Others & $\mathrm{NdgR}$ & S. coelicolor $\mathrm{A} 3(2)$ & $(\mathrm{G} / \mathrm{T}) \mathrm{T}(\mathrm{C} / \mathrm{T}) \mathrm{CAC}(\mathrm{C} / \mathrm{A})(\mathrm{C} / \mathrm{T} / \mathrm{A})(\mathrm{T} / \mathrm{C})(\mathrm{G} / \mathrm{C})(\mathrm{T} / \mathrm{C}) \mathrm{GGAC}$ & 178 \\
\hline TF & Others & WblC & S. coelicolor A3(2) & GAAAWY & 179 \\
\hline TF & Others & NsrR & S. coelicolor A3(2) & AASRMGMAYMTVRBMTVCSMRTT & 180 \\
\hline
\end{tabular}

Bold motif sequences were not indicated in the paper but were derived from the motif figure. ${ }^{a}$ SF, sigma factor. ${ }^{b} \mathrm{TF}$, transcription factor. ${ }^{\mathrm{c}} \mathrm{D}, \mathrm{S}$, development and secondary metabolism. ${ }^{\mathrm{d}} \mathrm{D}$, development. e $\mathrm{S}$, secondary metabolism. 
transcription factors are the atypical response regulators BldM and Whil, which orchestrate two different regulons depending on their dimerisation partner. ${ }^{163}$ The BldM homodimer controls genes whose functions are involved in aerial hyphae, spore formation, and 2-methylisoborneol biosynthesis, ${ }^{164}$ whereas the BldM-Whil heterodimer controls genes whose functions are involved in spore maturation and type II polyketide biosynthesis. These results suggest that binding partners and the corresponding mode of action should be investigated to elucidate the differential regulons in addition to ChIP-seq. In the case of the pleiotropic response regulator MtrA of the twocomponent system MtrAB, two independent ChIP-seq experiments were performed in two species. ${ }^{165,166}$ While the genes involved in the regulation of growth and sporulation were detected as the regulon in common, the genes involved in the secondary metabolism were differentially detected in each species. MtrA activates ACT and RED biosynthetic genes in $S$. coelicolor $A 3(2)$, but it represses chloramphenicol biosynthetic genes in S. venezuelae NRRL B-65442, reflecting the necessity of independent ChIP-seq experiments in different species, particularly in terms of secondary metabolism. ChIP-seq analysis of the two similar pleiotropic transcription factors ScbR and ScbR2 in S. coelicolor A3(2) detected many distinct targets encompassing diverse cellular processes. They formed complex regulatory cascades, sub-networks, and feed forward loops with their targets to control the development and antibiotic production to respond to fluctuating environmental signals. This intricate crosstalk between the two regulators provided a deeper understanding of the tight regulation of SM production. ${ }^{167}$ Taken together, ChIP-seq of these pleiotropic transcription factors revealed that they are located at the upper hierarchical position of the complex transcription network and tightly connect the development and secondary metabolism in response to diverse cellular status by changing the mode of action of their DNA-binding.

In general, pathway-specific transcription factors are located within their target smBGC. ${ }^{181}$ However, in S. albus S4, identification of binding sites of CSR FscRI located in the candicidin smBGC using ChIP-seq revealed that FscRI also binds to the promoter of the antimycin smBGC to control both the candicidin and antimycin smBGCs. ${ }^{169}$ In other words, FscRI coordinately controls the biosynthesis of both SMs, which is the first observation of cross-regulation of disparate smBGCs. Some transcription factors that control secondary metabolism exist outside of smBGCs. For example, CagR of the two-component system CagRS has 162 target sites, mainly involved in CA biosynthesis and primary metabolism related to precursor biosynthesis. ${ }^{170}$ This study indicated that CagRS is a pleiotropic regulator that effectively controls the whole biosynthetic processes of CA in S. clavuligerus F613-1. Interestingly, nucleoid-associated protein Lsr2 in S. venezuelae ATCC 10712, which is homologous to $\mathrm{H}-\mathrm{NS}$ in $E$. coli, binds to AT-rich sequences across the genome and broadly represses the transcription of several smBGCs. Gehrke et al. demonstrated the production of new metabolites by manipulating Lsr2 activity, which can be a potential engineering target for the activation of silent smBGCs. ${ }^{171}$ As these genes are mainly involved in
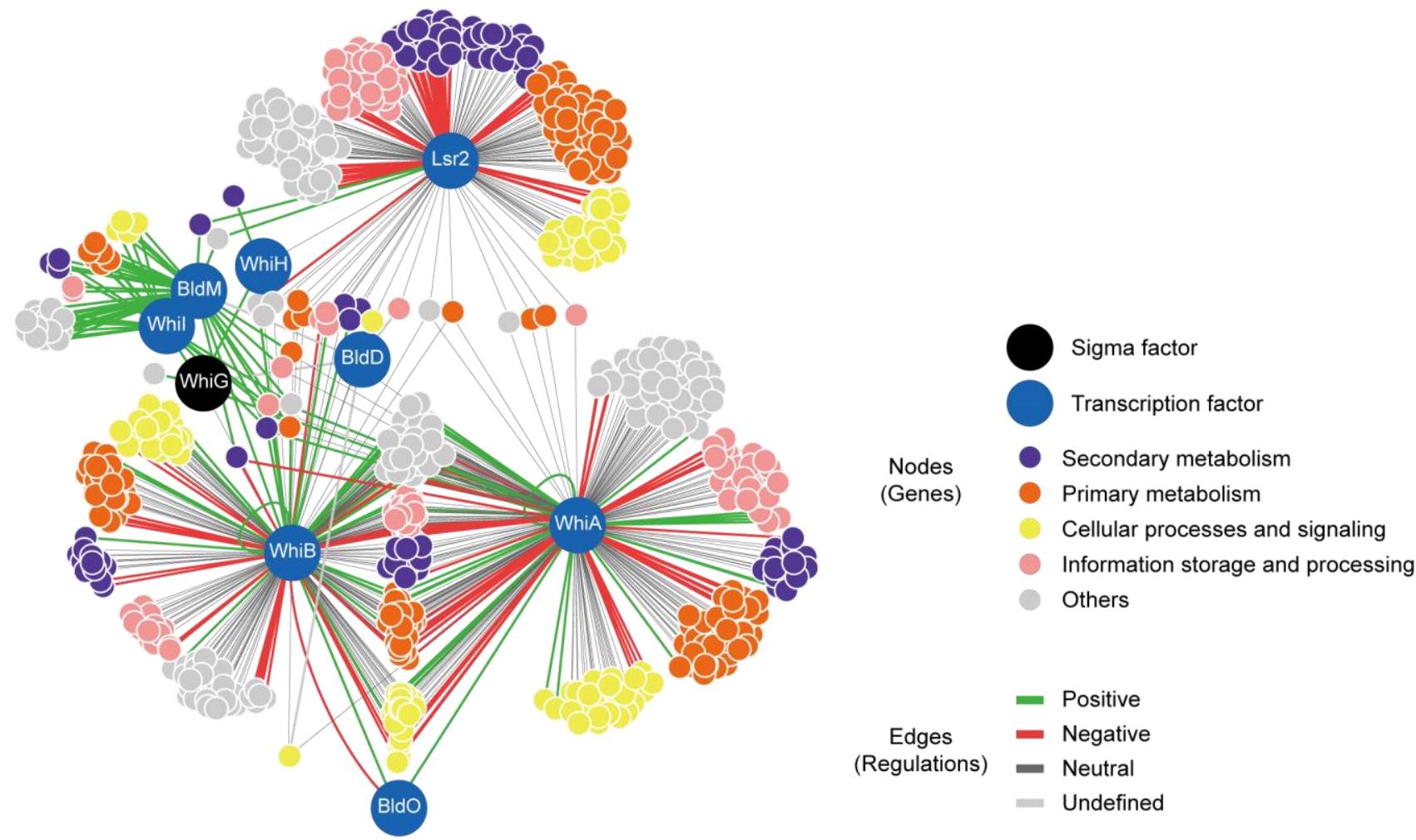

Fig. 10 Regulatory network of nine transcriptional regulators in S. venezuelae ATCC 10712 based on ChIP-seq results. Regulon of one sigma factor, WhiG, and eight transcription factors, including BldM, Whil, WhiH, Lsr2, WhiA, WhiB, BldD and BldO, were merged to construct the regulatory network. Direction of edge is from transcriptional regulators to linked genes. 
secondary metabolism, they may be ideal engineering targets for enhancing SM production.

Several transcription factors are related to developmental processes rather than secondary metabolism. For example, the BldC regulon comprises $>300$ genes, which mainly govern aerial mycelium formation and prevent premature sporulation. ${ }^{172}$ WhiA and WhiB also had >200 target genes, mainly for the initiation of sporulation septation and chromosome segregation, which were revealed to act cooperatively to control the expression of a common set of target genes. ${ }^{173,174}$ Moreover, ChIP-seq coupled with the structural and biochemical analysis of the master regulator BldD, which governs the repression of sporulation during vegetative growth, revealed that BldD dimerization is mediated by tetrameric c-di-GMP that regulates its DNA-binding activity. ${ }^{175,176}$ The regulons of $\mathrm{NdgR}^{178}$ and WbIC $^{179}$ were mainly enriched for the regulation of branchedchain amino acids and ribosome maintenance, respectively. However, these transcription factors might also have potential effects on secondary metabolism via direct control of poorly characterised genes or indirect control of interconnected regulatory networks. Moreover, a large proportion of Streptomyces transcription factors have not been investigated for their regulon. In this respect, ChIP-seq data of various transcription factors should be integrated for a holistic view of transcriptional regulation. For example, the regulatory network of nine transcription regulators in S. venezuelae ATCC 10712 was constructed by integration of their ChIP-seq data (Fig. 10). Along with the hierarchical regulatory structure between transcription factors, the network revealed that a large portion of genes are repressed by Lsr2. These genes are related to secondary metabolism, confirming the specialised function of Lsr2 toward secondary metabolism. Therefore, ChIP-seq data of various transcription factors should be further accumulated and integrated into the united database for a comprehensive understanding of the transcriptional regulatory network in Streptomyces.

\section{Translatomics: Elucidation of translational regulation of secondary metabolism}

SM production in Streptomyces is tightly regulated at the transcription level, as described above. Moreover, it is expected that additional regulation at post-transcriptional levels would affect gene expression. The most well-known example is the bldA gene, which encodes a unique tRNA that carries leucine corresponding to the TTA (UUA) codon, which is the rarest codon within the G/C-rich Streptomyces genomes. ${ }^{182}$ Thus, the translation level of TTA-containing genes is controlled by the expression level of the bldA gene. For example, the pleiotropic regulator AdpA and the aerial mycelium formation regulator $A m f R$ are known to be regulated by BldA to form a feedback loop to tightly control morphological differentiation. ${ }^{183}$ Also, the StrR of S. griseus IFO 13350, which is a transcriptional activator of streptomycin production, and Actll-ORF4 of $S$. coelicolor A3(2), which is a transcriptional activator of ACT production, contains TTA codons. ${ }^{184,185}$ In addition, the accumulation of guanosine tetraphosphate (ppGpp) promotes global expression changes including morphological differentiation and secondary metabolism. ${ }^{186,187}$ According to the ppGpp level, the open complex stability of RNA polymerase, sigma factor, and promoter could be altered, which controls transcription level, and ppGpp directly binds to the translational initiation factor to control the translational level. Furthermore, the Streptomyces genome possesses many RNases ${ }^{188,189}$ and riboswitches, ${ }^{190,191}$ which are potentially involved in posttranscriptional regulation.

This regulation cannot be elucidated with transcriptomic analysis alone. To elucidate the translational dynamics, ribosome profiling (Ribo-Seq) has been recently developed. ${ }^{192}$ This technique sequences ribosome protected footprints (RPFs), which represent the genome-wide positions of the translating ribosomes on mRNA transcripts. Ribosome profiling has been implemented in six Streptomyces species: S. coelicolor A3(2), S. avermitilis MA-4680, S. clavuligerus ATCC 27064, S. lividans TK24, S. venezuelae ATCC 15439, and S. tsukubaensis NRRL $18488 .{ }^{69,78,86,193}$ These studies revealed that (i) the increment of translation level of the secondary metabolism genes from early to late growth phases was not sufficient compared to the increment of their transcription level, which is referred to as translational buffering ${ }^{78}$; (ii) in S. coelicolor A3(2), several CSRs, including CdaR, Actll-ORF4, RedD, and RedZ, were translationally induced earlier than transcriptionally at transition phase ${ }^{78}$; and (iii) in S. lividans TK24 and S. clavuligerus ATCC 27064, the translation efficiency of ATG (AUG) start codon containing genes was higher than that of other start codon containing genes, suggesting potential regulation at translation initiation levels. ${ }^{69,86}$

Although the underlying mechanisms remain to be elucidated, they are suspected to be related to translational regulation by the stringent response, translation efficiency determined by $5^{\prime}$ UTR sequence, and ribosome pausing during translation. These points can be potential engineering targets to optimise SM production. Further ribosome profiling experiments of Streptomyces species under various conditions are essential to demonstrate the underlying mechanisms. To accelerate this, several technical limitations should be considered, including ribosome arrest by the flash-freezing method, and a wider range of RPF size selection will increase the reproducibility and reduce the technical bias of ribosome profiling. ${ }^{194,195}$ Another challenge of ribosome profiling is to distinguish between the RPFs that are involved in active translation and those in which the ribosomes are paused. Recently reported disome profiling techniques, which recover only the disome rather than the monosome to enrich the footprint of translational pausing, can be a breakthrough for dissecting the underlying translational mechanisms in Streptomyces. ${ }^{196,197}$

\section{Proteomics: Understanding secondary metabolism and discovery of natural products in Streptomyces}

Ribosome profiling enables the inference of translational regulation and protein abundance, but the presence of post- 
translational modifications (PTMs) is hampered. Indeed, assessing the protein turnover rates using SILAC and ITRAQ label-chase strategies in S. coelicolor A3(2) showed significant diversity of protein turnover rates, even among highly abundant housekeeping proteins, highlighting the need for direct measurement of protein levels. ${ }^{198}$ Likewise, the discordance between mRNA and protein levels was reported in S. coelicolor A3(2). The majority of genes encoding RNA polymerase, housekeeping sigma factors, and ribosome proteins showed a sharp decrease in mRNA levels in the stationary phase, but not for protein level. ${ }^{199}$ This divergent mRNA-protein relationship was correlated with the codon usage, RBS strength, and degradation rate, reflecting the presence of complex translational regulatory elements. ${ }^{200}$ To this end, proteomics is essential to directly measure the actual cellular protein profiles for understanding SM production, which is interconnected with numerous regulatory systems.

In this review, we organised proteomics studies of Streptomyces according to two main categories: (i) comparative proteomics for understanding and identifying potential targets for enhancing SM production, and (ii) targeted proteomics for the discovery of novel SMs and their link to smBGCs. The former category was then subcategorised according to the sample types, which were obtained from different conditions, genetic perturbations, and PTMs. The latter category was subcategorised according to proteomic strategies, which
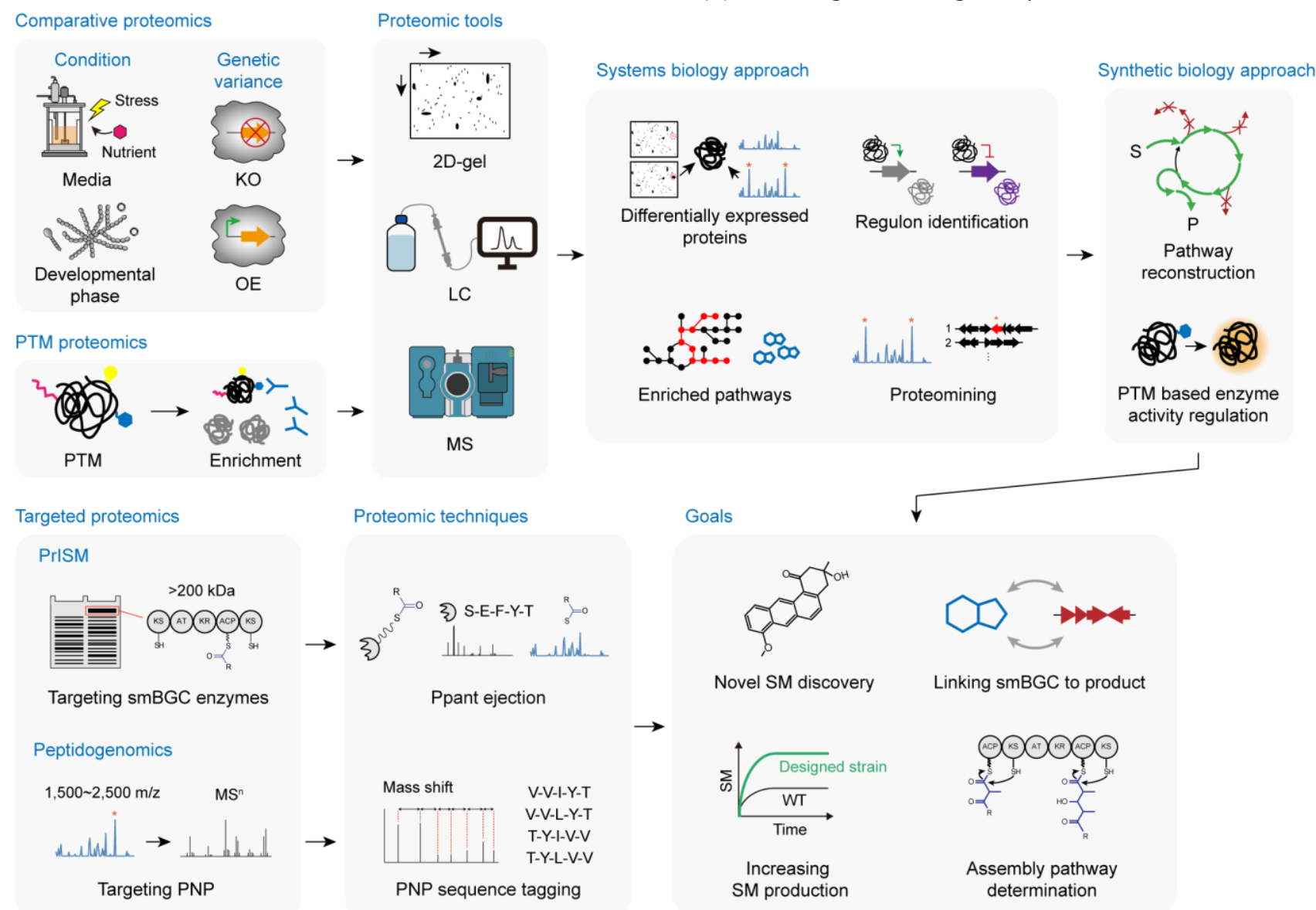

include proteomining, Proteomic Investigation of Secondary Metabolism (PrISM), and peptidogenomics (Fig. 11).

\subsection{Comparative proteomics for understanding and identifying potential targets for enhancing SM production}

The most abundant proteomic approach is bottom-up untargeted quantitative proteomics, which is a comparative analysis of protein abundance between different samples. ${ }^{201}$ First, comparative proteomics between the samples at the different developmental phases showed that SM production coincides with morphological differentiation. For example, proteomic analysis of $S$. coelicolor $\mathrm{A} 3(2)$ at the $\mathrm{MI}$ phase (compartmentalised mycelium) and MII phase (multinucleated hyphae) revealed that the metabolic switch from primary to secondary metabolism occurred between the $\mathrm{MI}$ and MII phases. Accordingly, the level of ACT biosynthetic enzymes increased. ${ }^{202}$ Many pleiotropic regulators involved in both morphological transcription and secondary metabolism, especially bld and whi genes, account for this metabolic switch, being regarded as potential engineering targets for enhancing SM production. Second, comparative proteomics of the samples in different media compositions revealed a change in the expression of pathways related to secondary metabolism in response to the key metabolite. For instance, proteomic analysis of tryptophan-supplemented conditions in S. coelicolor $A 3(2)$ showed global changes in protein abundance, including

Fig. 11 Overview of proteomics in Streptomyces. KO: knockout; OE: overexpression; LC: liquid chromatography; MS: mass spectrometry; PTM: post translational modification; PNP: peptide natural products. 
primary metabolism, morphological development, and secondary metabolism, resulting in the production of CDA and ACT. ${ }^{203}$ The increased production occurred not only because tryptophan is their direct precursor but also because tryptophan induces the transcription of trp genes, resulting in an increase in the SM biosynthetic enzymes and other global effects. Other factors such as $\mathrm{pH}$ shock ${ }^{204,205}$ and pitching ratio $^{206}$ could affect SM production with global proteomic changes.

Meanwhile, genetic perturbation often leads to global proteomic changes, resulting in phenotypic changes, including SM production. In this respect, comparative proteomics allow us to understand gene function and identify potential targets for enhancing SM production. For example, comparative proteomics of a single knockout of the cephamycin $\mathrm{C}$ and clavulanic acid supercluster regulator CcaR in the S. clavuligerus ATCC 27064 strain showed a reduced expression of supercluster genes, which confirmed that CcaR positively regulates the production of the two SMs. ${ }^{207,208}$ Pathway-specific regulators as well as genes with global function can influence SM production. In the case of S. lividans TK24, a polyphosphate kinase gene ( $p p k$ ) single-knockout strain resulted in the dramatic overproduction of ACT compared to the wild type, and subsequent comparative proteomics studies revealed a link between lipid metabolism and ACT biosynthesis. ${ }^{209}$ In addition, comparative proteomics of S. coelicolor $\mathrm{A} 3(2)$ and a sigN single knockout strain revealed that SigN controls ACT biosynthesis-related genes including putative oxidoreductase and branched-chain $\alpha$-keto acid dehydrogenase, indicating that secondary metabolism is linked with the stress responses. ${ }^{210}$

Comparative proteomics studies have also been conducted on the Streptomyces strains harboring multiple or unexpected genetic variances, leading to the differential production of the SM, to understand the metabolic change and find the key genes for high SM production. For example, among the streptomycin resistant mutant of $S$. coelicolor, the KO-179 strain overproduced ACT compared to the wild type. ${ }^{211}$ Comparative proteomic analysis of the two strains identified the significant overexpression of the $S$-adenosylmethionine (SAM) synthetase gene (metK) in the mutant strain with increased SAM level. Based on this result, metK was additionally overexpressed in the mutant, resulting in the further improvement of ACT production. Another representative example is the comparative proteomics on the doxorubicin less-producing mutant strain of $S$. peucetius ATCC $27952 .{ }^{212}$ To increase the precursor CoA pool to support the doxorubicin production, the pantothenate kinase gene (panK) of E. coli was introduced into S. peucetius, however, doxorubicin production was instead decreased unexpectedly. Comparative proteomics revealed global proteomic changes including the decreased expression level of efflux proteins DrrAB of panK-integrated S. peucetius. By the overexpression of $\operatorname{dr} A B$, the secretion of doxorubicin was enhanced, resulting in a 2-fold increase of doxorubicin production. All of these examples showed the strategies of identifying potential targets for enhancing the SM production using comparative proteomics.

The most significant advantage of proteomics is that it detects PTMs of proteins. ${ }^{213}$ PTMs either promote or inhibit protein functions, and they are generally reversible, which is beneficial to rapidly regulate protein activity in response to dynamic cellular status. ${ }^{214}$ PTMs of several proteins have been analysed using specific antibodies that recognise PTM residues, revealing the global distribution of various PTMs in Streptomyces proteins. ${ }^{215-217}$ Among them, several examples showed that PTMs are closely related to secondary metabolism. A representative example is the acetylome analysis in $S$. griseus IFO 13350.214 Among the 134 identified acetylated proteins, a deoxysugar epimerase, StrM, involved in streptomycin biosynthesis, was highly acetylated at its Lys70 residue, which is critical for its enzymatic activity. In S. coelicolor A3(2), time series phosphoproteome analysis identified approximately 100 phosphorylation sites from the total proteins, and half of them changed their phosphorylation state depending on the time series. ${ }^{218}$ Notably, the phosphorylation of a key bacterial cell division protein, FtsZ, resulted in reduction of sporulation and ACT production. Another example was crotonyl-proteomics in $S$. roseosporus, which revealed $>1,000$ crotonylation sites. ${ }^{219}$ Among these numerous targets, the crotonylation of glucose kinase Glk inhibited its catalytic activity reversibly through the growth phases, which was the key determinant for the phenotypic changes of delayed aerial mycelium development and postponed secondary metabolism. As these three examples show, most PTMs have negative effects on SM production. Proteomics for pupylated proteins in $S$. coelicolor $A 3(2)$ suggested positive effects of pupylation on secondary metabolism. ${ }^{220}$ The effects of other PTMs, such as glycosylation and succinylation on secondary metabolism should be further investigated to determine the potential engineering targets for enhancing SM production.

\subsection{Targeted proteomics for the discovery of novel SMs and linking to smBGCs}

Another important use of the proteomic approach is to discover novel SMs and link to its smBGCs through streamlined strategies such as proteomining, PrISM, and peptidogenomics. ${ }^{221} \mathrm{MS}$ based proteomics pipelines of these strategies not only identify the biosynthetic machinery but also provide simultaneous identification of the end product by integration with metabolomics.

Proteomining is the bottom-up strategy to identify smBGC and its products by the combination of quantitative proteomics and metabolomics. ${ }^{222}$ Based on this, novel compounds and their smBGCs were characterised, including an antimicrobial compound, juglomycin; ${ }^{222}$ the antibiotic, 7-prenylisatin; 223 and the catecholate hydroxamate siderophores, qinichelins. ${ }^{224}$ The other two strategies used top-down proteomic approaches to separate the target proteins first, and then analyse them subsequently. The PrISM pipeline permits targeted proteomic analysis of the two most important classes of biosynthetic enzymes, PKS and NRPS. The peptide mixture was subjected to LC-MS/MS to detach the 4'-phosphopantetheine (Ppant) arm by thermal activation using infrared multiphoton dissociation 
Comparative metabolomics

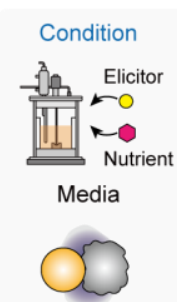

Co-culture

Metabolomic tools

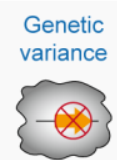

$\mathrm{KO}$

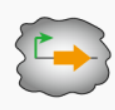

OE

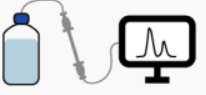

LC

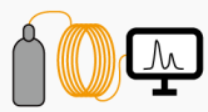

GC

Untargeted metabolomics

Newly isolated strains

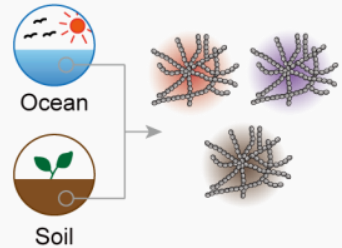

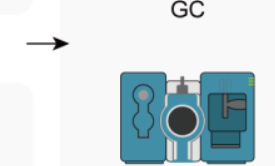

MS

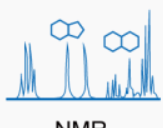

Systems biology approach Key metabolite identification

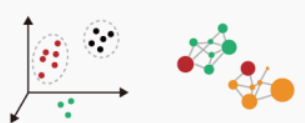

MVDA

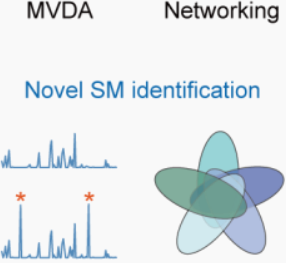

Metabolic profiling
Synthetic biology approach

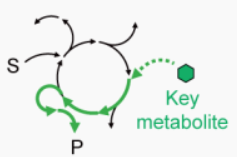

Media optimization

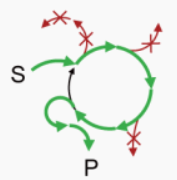

Strain optimization
Goals

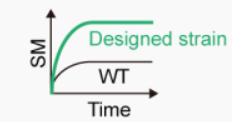

Increasing SM production

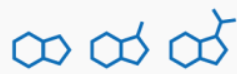

SM derivatives

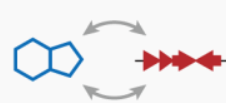

Linking smBGC to product

Fig. 12 Overview of metabolomics in Streptomyces. KO: knockout; OE: overexpression; LC: liquid chromatography; GC: gas chromatography; MS: mass spectrometry; NMR: nuclear magnetic resonance.

(IRMPD) or collision-induced dissociation (CID).225,226 The Ppant arms could contain the peptides of acyl and peptidyl carrier proteins of PKS and NRPS, with the thioester linkage to the PK and NRP intermediates, respectively. After identifying the corresponding peptides of PKS and NRPS, degenerate PCR was used to identify the genomic position of the corresponding enzymes. The cognate SM can be identified by annotationbased analysis of the PKS and NRPS with the integration of subsequent metabolomics. PrISM led to the discovery of many SMs and linking to their smBGCs in Streptomyces, including phosphinothricin tripeptides; ${ }^{226}$ mixed-ligand siderophores, gobichelin $\mathrm{A}$ and $\mathrm{B}^{227}$ anti-proliferative flavopeptins; ${ }^{227}$ and threonine-containing siderophore-like compounds. ${ }^{228}$ For a similar proteomic approach, the orthogonal active site identification system (OASIS) can characterise PKS and NRPS using activity-based probes, ${ }^{229}$ but has limitations in the range of detectable domains. However, PrISM is restricted to PKS and NRPS and requires the prediction of SmBGC products based on databases. To compensate for this, PK and NRP intermediates attached to the Ppant arm can be analysed together using MS, identifying the domain substrate of each module to experimentally 'debug' the biosynthetic pathway of PK and NRP having chain elongation in a modular manner. ${ }^{230}$ Peptidogenomics, which is a chemotype-to-genotype genome mining approach, directly detects RiPPs and NRPs by tandemMS to obtain de novo sequence tags. ${ }^{231}$ The sequence tag information is matched with the sequence of the precursor peptide encoding gene for RiPPs and the predicted product sequence for NRP across the genome to identify the corresponding smBGC. Using peptidogenomics, novel natural products were discovered with their smBGC, such as the antimicrobial, stendomycin; ${ }^{231}$ the anti-infective, arylomycin; ${ }^{232}$ linaridin RiPP, legonaridin; ${ }^{233}$ and other analogues of stenothricin. ${ }^{234}$
For successful application of proteomics, the accumulation of multi-omics data and biochemical information is required for the identification and analysis of proteins. Functional analysis of the proteins, including structural assays, bioactivity assays, and mechanistic studies, should also be performed at the system scale. For novel SM discovery, proteomics can be utilised in retro-biosynthetic modular engineering by identifying the substrate of each domain in PKS and NRPS, through combined analysis with a Ppant ejection assay and NMR analysis, and rationally connecting each module to synthesise non-native novel products. ${ }^{235}$

\section{Metabolomics: Understanding dynamic metabolite profiles from primary to secondary metabolism and identifying novel SMs}

Metabolomics is the most essential technique for SM research, which identifies and quantifies low molecular weight metabolites in a global manner. ${ }^{236,237}$ Moreover, whole metabolic flux can be analysed by comparative metabolomics in various conditions and genetic backgrounds, enabling the understanding of interconnected pathways with secondary metabolism and identifying key metabolites for enhancing SM production in Streptomyces (Fig. 12). Statistical analysis methods have been discussed in comprehensive reviews. ${ }^{238,239}$

Dereplication of MS spectra is an important step for the identification of metabolites based on various databases and software such as Cycloquest, Global Natural Product Social (GNPS), and Dictionary of Natural Products (DNP). ${ }^{238}$ In addition, molecular networking of MS spectra based on the MS/MS database GNPS platform, enabled correlation analysis between metabolites and dereplication of both known and unknown metabolites by constructing a network of nodes (each metabolite) and edges (correlation between spectra). ${ }^{240,241}$ This allows metabolomics to discover novel metabolites. 
Metabolomics data is the main output of the 'test' part of the DBTL cycle in synthetic biology to understand and learn from high-throughput screening and measurement of the engineering result. ${ }^{242}$ Additionally, metabolomics data reflects the phenotype of genomic, transcriptomic and proteomic networks to fill the gap between the genomic ability and the actual SM production. ${ }^{243,244}$

Streptomyces metabolomic studies have been extensively reported compared to other omics studies, and they were categorised into (i) understanding or identifying potential targets for enhancing SM production, and (ii) discovery of novel SMs and linking to smBGC. These categories were subcategorised according to the sample types, which are different conditions, genetic perturbation, and newly isolated strains. Over the last decade, about a hundred metabolomics studies of Streptomyces have been reported. Most of them discussed specific SMs, whereas some addressed taxonomic classifications, modelling, and other topics (Fig. 13A). Among the SM related studies, the 'discovery' category was more abundant than the 'understanding' category, reflecting the importance of metabolomics in the field of novel SM discovery. The number of annually reported studies showed that all categories and subcategories have been evenly reported over the last decade with an increasing trend (Fig. 13B). For the distribution of Streptomyces strains, the majority of studies in both categories were mostly focused on the new strains (Fig.
13C). This was due to the high proportion of novel SMs discovered in the newly characterised strains. Next, SMs discussed in the metabolomics studies of each category were divided by their types (Fig. 13D). For the 'discovery' category, NRP was highly abundant, whereas PK and PK-NRP were less abundant. Notably, terpene and alkaloid SM types showed relatively higher proportions in the 'discovery' category, suggesting that the metabolomics approach is favourable for the direct discovery of novel SMs even without genomic information.

\subsection{Understanding and identifying potential targets for enhancing SM production}

As SMs are generally known to be produced at the later growth phase, time series metabolomics was used to understand the dynamics of SM production. For example, metabolic profile analysis under different morphologies of S. hygroscopicus U1$6 E 7$ revealed that sufficient oxygen supply of the longer filament cell is the main factor for higher rapamycin production, exhibiting a connection between morphology and secondary metabolism. ${ }^{245}$ As another example, time series metabolomics of $S$. nodosus identified the key metabolites for higher production of amphotericin B in the stationary phase, which are involved in oxygen-uptake, precursor-acquiring, and productexporting pathways. Based on these results, amphotericin B
A

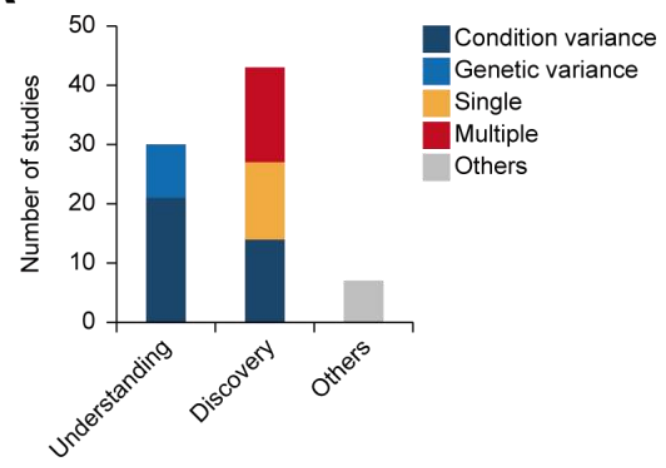

C

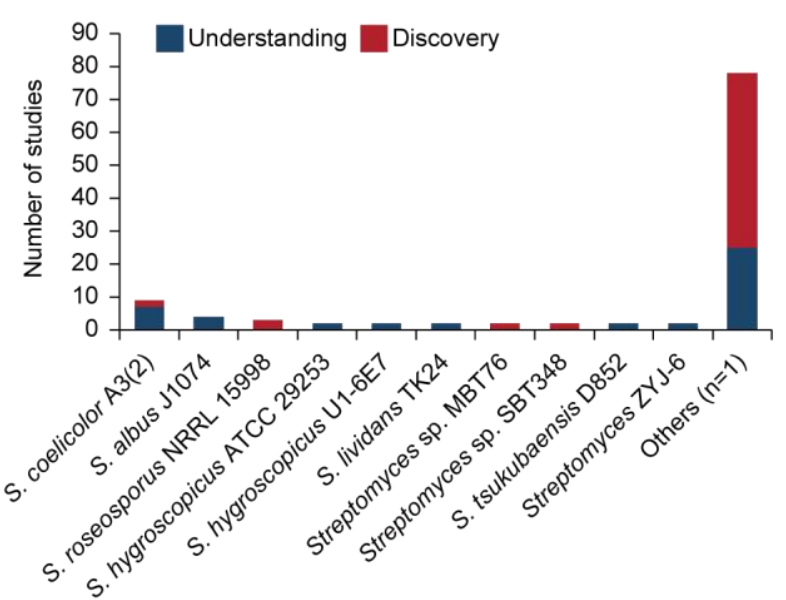

B

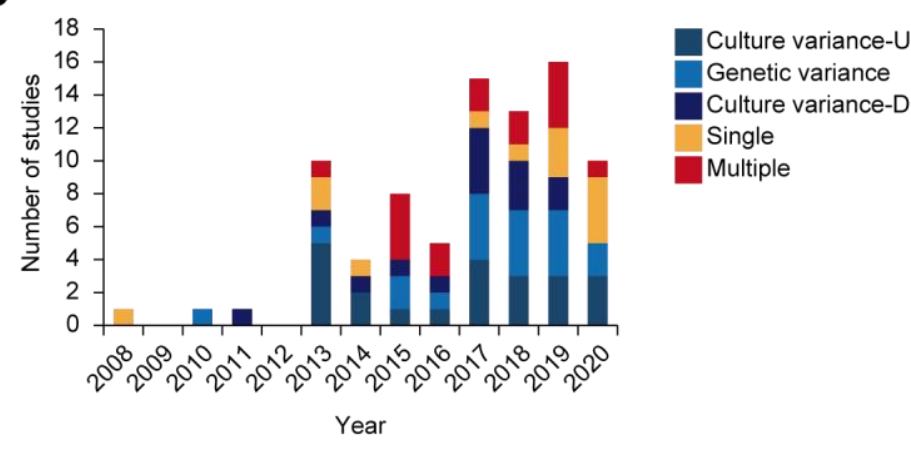

D

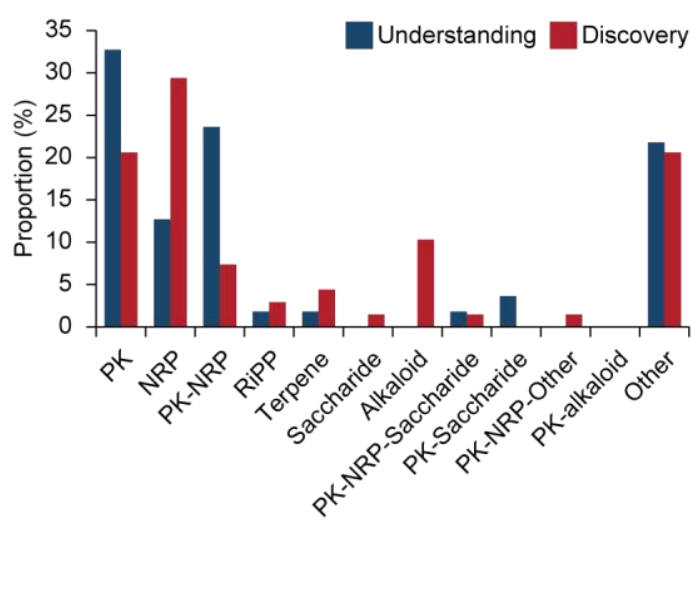

Fig. 13 Current status of metabolomics studies in Streptomyces. (A) Distribution of the category of metabolomics studies, (B) Number of annually reported metabolomics studies, (C) Number of Streptomyces strains in metabolomics studies, (D) Number of SM types in metabolomics studies. 
production was further enhanced by the overexpression of key enzymes. ${ }^{246}$

The effects of supplemented media compositions or exogenous feeding metabolites on SM production were also analysed by comparative metabolomics. For instance, SAM supplementation of $S$. avermitilis culture is known to increase avermectin production. Comparative metabolite profiling between the cultures with and without SAM revealed the mechanism of this positive effect. ${ }^{247}$ It was found that SAM supply led to an increase in the concentration of precursor metabolites, so the co-feeding strategy of glucose and SAM further improved the avermectin production. In addition, comparative metabolomics in more complex conditions including combinations of different media and time series enabled the deeper understanding of the SM production. For example, molecular networking analysis of the metabolomes from different media and growth phases revealed dynamic changes in lipid composition in S. coelicolor A3(2). ${ }^{248}$ Specifically, ornithine lipid was found to accumulate under phosphate limitation and later growth phases, and deletion of its biosynthetic genes showed precocious morphological development and ACT production, reflecting the connections between secondary metabolism and lipid metabolism. Indeed, other culture conditions, including chemical elicitors, ${ }^{249,250}$ $\mathrm{pH},{ }^{251}$ and co-culture, ${ }^{252,253}$ have been reported to change SM production, and their key metabolites were revealed by metabolomics.

Comparative metabolomics revealed the effects of genetic perturbation on global metabolite profiles, which ultimately led to changes in SM production. For example, metabolite profiling of single knockout or overexpression strains of $h r m A, h r m B$ and $\mathrm{hrmH}$ in the hormaomycin BGC revealed not only the changes in hormaomycin quantity but also shifting to derivatives. ${ }^{254}$ This result implied that metabolomics can detect the intermediates and analogues of the SM with very similar structures, which cannot be predicted by other omics data. In addition, the identification of key metabolites in non-, less-, and highproducing strains is a frequent strategy for understanding and enhancing SM production. Recently, metabolite profiling of the ACT overproducing strain HYO1 of S. coelicolor A3(2) revealed that the dynamic degradation of triacylglycerol mobilised the triacylglycerol pool, resulting in an increase in polyketide biosynthesis ${ }^{255}$. Additional induction of fatty acyl-CoA synthetase increased the triacylglycerol pool and enhanced the production of many polyketides such as ACT, jadomycin B, oxytetracycline, and avermectin. Taken together, key metabolites or target genes for enhancing SM production were effectively identified by comparative metabolomics. Through integration with other omics data, metabolomics would enable a more comprehensive understanding of secondary metabolism.

\subsection{Discovery of novel SMs and linking to smBGCs}

Metabolomics is highlighted for its ability to directly identify novel SMs. The main hurdles for the discovery of novel SMs are (i) difficulty in metabolite detection owing to its low production under laboratory conditions and (ii) rediscovery of known SMs and difficulty with dereplication. ${ }^{256,257}$ Metabolomics has prominent advantages in overcoming these hurdles in terms of (i) quantitative detection with high resolution for low abundant metabolites from untargeted metabolomics of different samples and (ii) systematic dereplication of unknown metabolites and chemical structure prediction by molecular networking based on metabolome databases. We subdivided the metabolomics studies of novel SM discovery according to the following approaches: (i) comparative metabolomics under different conditions and (ii) untargeted metabolite profiling of a single strain or multiple strains isolated from a specific environment or showing bioactive phenotypes.

Many novel SMs have been detected from the metabolomics of previously known strains by the activation of silent BGCs through variations in culture conditions such as growth phases, media compositions ${ }^{258,259}$, stresses ${ }^{260,261}$, elicitors ${ }^{262}$ and co-culture. ${ }^{263,264}$ For instance, the isocoumarin producing Streptomyces sp. MBT76, which has antimicrobial activity against ESKAPEE pathogens, is an example of a novel SM discovery using time-series metabolomics. ${ }^{265}$ In addition, the methoxylation moiety of isocoumarins is critical for antimicrobial activity, showing the capability of metabolomics to identify the important modification of the SM for bioactivity. A representative example of comparative metabolomics under different media compositions is the one-strain many compounds (OSMAC) approach in Streptomyces sp. MBT27, which revealed novel 4-quinazolinnone alkaloid in high glycerol content medium. ${ }^{258}$ With regard to stress conditions, metabolomics approaches under nickel stress condition identified novel antimicrobial cyclizidine analogues in Streptomyces sp. WU20, ${ }^{260}$ and novel antibiotics stremycin A and B in S. pratensis NA-ZhouS1, ${ }^{261}$ respectively. Recently, the high-throughput metabolomic strategy for the discovery of novel canucin A and B in S. canus NRRL B3980 was reported. The treatment of about 500 elicitor libraries promoted differential stress responses in 96 well plates, and then high-throughput MS screening of each well was performed by laser-ablation-coupled electrospray ionization MS (LAESI-MS) for the detection of the novel MS spectra. ${ }^{262}$ Application of this strategy to other Streptomyces is expected to lead to the high-throughput discovery of the various novel SMs. Collectively, the metabolomics approach was effective for novel SM identification from silent smBGCs by quantitative detection of low abundant metabolites in differential conditions.

Untargeted metabolite profiling and subsequent dereplications using manual matching or molecular networking of a single strain or multiple strains are alternative approaches for the discovery of novel SMs. As an example of single strain studies, untargeted metabolomics coupled with the GNPS molecular networking for dereplication of the novel SMs discovered novel analogues of pumilacidins and surfactins from the Streptomyces sp. S063 CGMCC 14582 with anti-complement activities, ${ }^{266}$ and 2,5-diketopiperzaines from the marine-derived S. tendae VITAKN. ${ }^{267}$ Moreover, metabolomics coupled with molecular networking was also used for the identification of a bioactive strain and its novel SMs among multiple strains isolated from the same environment. For example, comparative 
metabolomics of six marine-isolated S. aculeolatus MA4 strains followed by PLA, PLS-DA and GNPS molecular networking identified unique MS/MS spectra of each strain, which were novel analogues of napyradiomycin and marinone. ${ }^{268} \mathrm{~A}$ recent example is the large-scale comparative metabolomics across 363 actinobacterial strains using a computational framework (BiG-SCAPE and CORASON) that identified novel detoxins. ${ }^{269}$

Although numerous metabolomics studies have been reported in Streptomyces to enhance SM production and discovery of novel SMs, further improvements are required in terms of high-throughput techniques and metabolome databases. ${ }^{236}$ The main technical bottleneck of metabolomics is the laborious experimental steps from the metabolite extraction to metabolite profiling by MS. In recent years, realtime and high-resolution imaging MS have been widely used to overcome these limitations. $252,253,262,270$ The application of Streptomyces cell-free systems for high-throughput screening of the SMs among different genetic backgrounds will also accelerate SM production engineering. ${ }^{271}$ Alternatively, a reporter system for Streptomyces could be developed, which is responsive to a specific SM for high-throughput screening without using MS. Enzymatic reaction based reporter systems such as GusA and BpsA have been utilised in Streptomyces, but they need specific substrates for their activity. ${ }^{272,273}$ Fluorescence based reporter systems such as sfGFP were reported in a few Streptomyces studies, 274-276 but autofluorescence and the diversity of the cell shape were challenges for application to high-throughput screening methods, such as FACS. Thus, an optimised pipeline for pre-treatment and usage of other fluorescent proteins with different excitation wavelengths from auto-fluorescence should be developed. In addition to the reporter system, a biosensing system in response to a specific SM should also be developed. Several biosensors have been used in Streptomyces, such as macrolide biosensing MphR277 and tetracycline biosensing TetR-family regulators. ${ }^{278}$ Another major challenge of metabolomics is the integration and expansion of metabolite databases. As described above, metabolite databases are essential for dereplication and annotation of each metabolite from MS spectra. However, databases for metabolomics are not currently universal compared to genomics and transcriptomics databases. Fortunately, the GNPS database is vigorously expanding by metabolomics data accumulation, and further integration of other databases, such as National Institute of Standards and Technology (NIST), and DNP, and their standardisation will enhance the accuracy of dereplication. The establishment of an optimised metabolomic pipeline for data processing, statistical analysis, and molecular networking, and the application of deep learning-based methods will also improve SM production.

\section{Concluding remarks}

Recently, paradigm shifts occurred in: 1) our systematic comprehension of secondary metabolism, 2) synthetic strain development for enhancing SM production, and 3) the development of a suite of molecular tools for Streptomyces.
Genomics unveiled the chemical diversity of Streptomyces that had been underestimated due to most smBGCs remaining silent under axenic culture conditions. Henceforth, various systematic approaches including transcriptomics, translatomics, interactomics, proteomics and metabolomics have been utilised to understand the complex regulation of secondary metabolism in Streptomyces. This review aimed to comprehensively describe the workflow of systematic approaches that have been developed for Streptomyces to date, with representative examples, and the state-of-the-art omics methods that have been or can be applied to Streptomyces. In addition, to obtain a systematic understanding to achieve a rational increase in SM production, finding engineering targets or developing genetic parts for engineering were discussed, which largely deployed knowledge from a systematic approach (Fig. 1).

The current limitation of systematic approaches to Streptomyces is the insufficiency of high-quality omics data within databases. However, reports of new discoveries by integrating a large amount of omics data have been increasing. In the case of E. coli, 45 different heterologous genes were expressed, individual transcriptome data were integrated, and independent component analysis was performed, revealing independently modulated gene sets (iModulon) that controlled together respond to heterologous gene expression. ${ }^{279}$ If the accumulated data is sufficient, this approach would be applicable to determine a common set of genes related to secondary metabolism in Streptomyces. In the case of proteomes and metabolomes, a large proportion of the data generated has not been deposited in a public database. Even in the case of deposited data, several repositories have been interchangeably used as databases, and integrated databases do not exist. A universal, integrated database for the proteome and the metabolome would be in high demand because it would be directly connected with the annotation accuracy of each protein and metabolite. In addition, the computational power and processing software to analyse enlarged data of MS spectral libraries obtained from different instrument platforms are indispensable for exploring novel SMs in a high-throughput manner. Building a full pipeline from data acquisition to comprehensive interpretation of proteomics and metabolomics would greatly advance the discovery of SMs.

To realize rational engineering for enhancing SM production based on systematic understanding, highly efficient synthetic biology tools should be practicable in Streptomyces in parallel. CRISPR/Cas-based genome-engineering, CRISPR/Cas-based transcriptional regulations, DNA synthesis and assembly techniques for large smBGCs, chassis strain development for heterologous expression, biosensors, genetic circuits and enzyme engineering are beginning to be intensively utilised for Streptomyces research. ${ }^{148,280}$ Eventually, systems and synthetic biology approaches for Streptomyces will be integrated into the DBTL cycle, providing a mutualistic interaction between the accumulation of knowledge and development of techniques required for the retrobiosynthesis of unnatural products.

\section{Author contributions}


Yeo Joon Yoon, Min-Kyu Oh, Bernhard O. Palsson, and ByungKwan Cho conceived and supervised the manuscript. Namil Lee, Soonkyu Hwang, and Byung-Kwan Cho analysed the data. Namil Lee, Soonkyu Hwang, Woori Kim, Yongjae Lee, Ji Hun Kim, Suhyung Cho, Hyun Uk Kim, Yeo Joon Yoon, Min-Kyu Oh, Bernhard O. Palsson, and Byung-Kwan Cho wrote the manuscript. All authors proofread the entire manuscript and provided suggestions for improvement on all sections.

\section{Conflicts of interest}

The authors declare no conflicts of interest.

\section{Acknowledgements}

This work was supported by the National Research Foundation of Korea (NRF) grant funded by the Korean government (MSIT) (2018M3A9F3079664 to B.-K.C., 2018 M3A9F3079662 to M.K.O., and 2019R1A2B5B03069338 to Y.J.Y). This research was also supported by the Collaborative Genome Program of the Korea Institute of Marine Science and Technology Promotion (KIMST) funded by the Ministry of Oceans and Fisheries (MOF) (No. 20180430 to Y.J.Y). This work was also supported by a grant from the Novo Nordisk Foundation (NNF10CC1016517 to B.O.P)

\section{References}

1. D. J. Newman and G. M. Cragg, Journal of Natural Products, 2016, 79, 629-661.

2. A. Craney, S. Ahmed and J. Nodwell, J Antibiot (Tokyo), 2013, 66, 387-400.

3. N. Lee, W. Kim, S. Hwang, Y. Lee, S. Cho, B. Palsson and B. K. Cho, Sci Data, 2020, 7, 55.

4. A. Ebrahim, E. Brunk, J. Tan, E. J. O'Brien, D. Kim, R. Szubin, J. A. Lerman, A. Lechner, A. Sastry, A. Bordbar, A. M. Feist and B. O. Palsson, Nat Commun, 2016, 7, 13091.

5. D. D. Marshall and R. Powers, Progress in Nuclear Magnetic Resonance Spectroscopy, 2017, 100, 1-16.

6. B. C. Blum, F. Mousavi and A. Emili, Mol Omics, 2018, 14, 307319.

7. F. Malpartida and D. A. Hopwood, Nature, 1984, 309, 462-464

8. F. Pojer, S. M. Li and L. Heide, Microbiology, 2002, 148, 39013911.

9. S. D. Bentley, K. F. Chater, A. M. Cerdeno-Tarraga, G. L. Challis, N. R. Thomson, K. D. James, D. E. Harris, M. A. Quail, H. Kieser, D. Harper, A. Bateman, S. Brown, G. Chandra, C. W. Chen, M. Collins, A. Cronin, A. Fraser, A. Goble, J. Hidalgo, T. Hornsby, S. Howarth, C. H. Huang, T. Kieser, L. Larke, L. Murphy, K. Oliver, S. O'Neil, E. Rabbinowitsch, M. A. Rajandream, K. Rutherford, S. Rutter, K. Seeger, D. Saunders, S. Sharp, R. Squares, S. Squares, K. Taylor, T. Warren, A. Wietzorrek, J. Woodward, B. G. Barrell, J. Parkhill and D. A. Hopwood, Nature, 2002, 417, 141-147.

10. H. Ikeda, J. Ishikawa, A. Hanamoto, M. Shinose, H. Kikuchi, T. Shiba, Y. Sakaki, M. Hattori and S. Omura, Nat Biotechnol, 2003, 21, 526-531.

11. N. Lee, S. Hwang, J. Kim, S. Cho, B. Palsson and B. K. Cho, Comput Struct Biotechnol J, 2020, 18, 1548-1556.

12. J. Harrison and D. J. Studholme, Microb Biotechnol, 2014, 7 , 373-380.
13. H. Musto, H. Naya, A. Zavala, H. Romero, F. Alvarez-Valin and G. Bernardi, Biochem Biophys Res Commun, 2006, 347, 1-3.

14. C. E. Singer and B. N. Ames, Science, 1970, 170, 822-825.

15. K. F. Chater and G. Chandra, J Microbiol, 2008, 46, 1-11.

16. J. L. Weissman, W. F. Fagan and P. L. F. Johnson, PLoS Genet, 2019, 15, e1008493.

17. P. Dyson and H. Schrempf, J Bacteriol, 1987, 169, 4796-4803.

18. J. N. Volff and J. Altenbuchner, Mol Microbiol, 1998, 27, 239 246.

19. F. A. Simao, R. M. Waterhouse, P. Ioannidis, E. V. Kriventseva and E. M. Zdobnov, Bioinformatics, 2015, 31, 3210-3212.

20. D. J. Studholme, Microb Biotechnol, 2016, 9, 3-7.

21. E. Zazopoulos, K. Huang, A. Staffa, W. Liu, B. O. Bachmann, K. Nonaka, J. Ahlert, J. S. Thorson, B. Shen and C. M. Farnet, Nat Biotechnol, 2003, 21, 187-190.

22. N. Ichikawa, M. Sasagawa, M. Yamamoto, H. Komaki, Y. Yoshida, S. Yamazaki and N. Fujita, Nucleic Acids Res, 2013, 41 D408-414.

23. S. A. Kautsar, K. Blin, S. Shaw, J. C. Navarro-Munoz, B. R. Terlouw, J. J. J. van der Hooft, J. A. van Santen, V. Tracanna, H. G. Suarez Duran, V. Pascal Andreu, N. Selem-Mojica, M. Alanjary, S. L. Robinson, G. Lund, S. C. Epstein, A. C. Sisto, L. K. Charkoudian, J. Collemare, R. G. Linington, T. Weber and M. H. Medema, Nucleic Acids Res, 2020, 48, D454-D458.

24. A. de Jong, S. A. van Hijum, J. J. Bijlsma, J. Kok and O. P. Kuipers, Nucleic Acids Res, 2006, 34, W273-279.

25. A. Starcevic, J. Zucko, J. Simunkovic, P. F. Long, J. Cullum and D. Hranueli, Nucleic Acids Res, 2008, 36, 6882-6892.

26. M. H. Li, P. M. Ung, J. Zajkowski, S. Garneau-Tsodikova and D. H. Sherman, BMC Bioinformatics, 2009, 10, 185.

27. T. Weber, C. Rausch, P. Lopez, I. Hoof, V. Gaykova, D. H. Huson and W. Wohlleben, J Biotechnol, 2009, 140, 13-17.

28. M. A. Skinnider, C. A. Dejong, P. N. Rees, C. W. Johnston, H. Li, A. L. Webster, M. A. Wyatt and N. A. Magarvey, Nucleic Acids Res, 2015, 43, 9645-9662.

29. M. H. Medema, K. Blin, P. Cimermancic, V. de Jager, P. Zakrzewski, M. A. Fischbach, T. Weber, E. Takano and R. Breitling, Nucleic Acids Res, 2011, 39, W339-346.

30. K. Blin, S. Shaw, K. Steinke, R. Villebro, N. Ziemert, S. Y. Lee, M. H. Medema and T. Weber, Nucleic Acids Res, 2019, 47, W81W87.

31. M. A. Skinnider, N. J. Merwin, C. W. Johnston and N. A. Magarvey, Nucleic Acids Res, 2017, 45, W49-W54.

32. P. Cimermancic, M. H. Medema, J. Claesen, K. Kurita, L. C. Wieland Brown, K. Mavrommatis, A. Pati, P. A. Godfrey, M. Koehrsen, J. Clardy, B. W. Birren, E. Takano, A. Sali, R. G. Linington and M. A. Fischbach, Cell, 2014, 158, 412-421.

33. G. D. Hannigan, D. Prihoda, A. Palicka, J. Soukup, O. Klempir, L. Rampula, J. Durcak, M. Wurst, J. Kotowski, D. Chang, R. Wang, G. Piizzi, G. Temesi, D. J. Hazuda, C. H. Woelk and D. A. Bitton, Nucleic Acids Res, 2019, 47, e110.

34. T. Weber and H. U. Kim, Synth Syst Biotechnol, 2016, 1, 69-79.

35. N. Ziemert, M. Alanjary and T. Weber, Nat Prod Rep, 2016, 33, 988-1005.

36. S. Lautru, R. J. Deeth, L. M. Bailey and G. L. Challis, Nat Chem Biol, 2005, 1, 265-269.

37. G. L. Challis and J. Ravel, FEMS Microbiol Lett, 2000, 187, $111-$ 114.

38. A. Vingadassalon, F. Lorieux, M. Juguet, G. Le Goff, C. Gerbaud, J. L. Pernodet and S. Lautru, ACS Chem Biol, 2015, 10, 601-610.

39. Q. Zhou, S. Ning and Y. Luo, Synth Syst Biotechnol, 2020, 5, 4958. 
40. L. Laureti, L. Song, S. Huang, C. Corre, P. Leblond, G. L. Challis and B. Aigle, Proc Natl Acad Sci U S A, 2011, 108, 6258-6263.

41. A. Thanapipatsiri, J. P. Gomez-Escribano, L. Song, M. J. Bibb, M. Al-Bassam, G. Chandra, A. Thamchaipenet, G. L. Challis and M. J. Bibb, Chembiochem, 2016, 17, 2189-2198.

42. W. Tao, A. Yang, Z. Deng and Y. Sun, Front Microbiol, 2018, 9, 1660.

43. Y. Tong, T. Weber and S. Y. Lee, Nat Prod Rep, 2019, 36, 1262 1280.

44. M. M. Zhang, F. T. Wong, Y. Wang, S. Luo, Y. H. Lim, E. Heng, W. L. Yeo, R. E. Cobb, B. Enghiad, E. L. Ang and H. Zhao, Nat Chem Biol, 2017, 13, 607-609.

45. Y. Zhao, L. Li, G. Zheng, W. Jiang, Z. Deng, Z. Wang and Y. Lu, Biotechnol J, 2018, 13, e1800121.

46. L. Li, K. Wei, G. Zheng, X. Liu, S. Chen, W. Jiang and Y. Lu, Appl Environ Microbiol, 2018, 84.

47. Y. Tong, P. Charusanti, L. Zhang, T. Weber and S. Y. Lee, ACS Synth Biol, 2015, 4, 1020-1029.

48. J. Tian, G. Yang, Y. Gu, X. Sun, Y. Lu and W. Jiang, Nucleic Acids Res, 2020, 48, 8188-8202.

49. J. Fontana, C. Dong, C. Kiattisewee, V. P. Chavali, B. I. Tickman, J. M. Carothers and J. G. Zalatan, Nat Commun, 2020, 11, 1618

50. J. H. Hu, S. M. Miller, M. H. Geurts, W. Tang, L. Chen, N. Sun, C. M. Zeina, X. Gao, H. A. Rees, Z. Lin and D. R. Liu, Nature, 2018, 556, 57-63.

51. S. E. Ongley, X. Bian, B. A. Neilan and R. Muller, Nat Prod Rep, 2013, 30, 1121-1138.

52. R. Liu, Z. Deng and T. Liu, Metab Eng, 2018, 50, 74-84.

53. M. Xu and G. D. Wright, J Ind Microbiol Biotechnol, 2019, 46, 415-431.

54. K. K. Nepal and G. Wang, Biotechnol Adv, 2019, 37, 1-20.

55. M. Komatsu, T. Uchiyama, S. Omura, D. E. Cane and H. Ikeda, Proc Natl Acad Sci U S A, 2010, 107, 2646-2651.

56. J. P. Gomez-Escribano and M. J. Bibb, Microb Biotechnol, 2011, 4, 207-215.

57. M. Myronovskyi, B. Rosenkranzer, S. Nadmid, P. Pujic, P. Normand and A. Luzhetskyy, Metab Eng, 2018, 49, 316-324.

58. Q. T. Bu, P. Yu, J. Wang, Z. Y. Li, X. A. Chen, X. M. Mao and Y. Q. Li, Microb Cell Fact, 2019, 18.

59. Y. Ahmed, Y. Rebets, M. R. Estevez, J. Zapp, M. Myronovskyi and A. Luzhetskyy, Microb Cell Fact, 2020, 19, 5.

60. H. Ikeda, J. Ishikawa, A. Hanamoto, M. Shinose, H. Kikuchi, T. Shiba, Y. Sakaki, M. Hattori and S. Omura, Nature Biotechnology, 2003, 21, 526-531.

61. M. Komatsu, K. Komatsu, H. Koiwai, Y. Yamada, I. Kozone, M. Izumikawa, J. Hashimoto, M. Takagi, S. Omura, K. Shin-ya, D. E. Cane and H. Ikeda, ACS Synth Biol, 2013, 2, 384-396.

62. J. P. Gomez-Escribano and M. J. Bibb, J Ind Microbiol Biotechnol, 2014, 41, 425-431.

63. M. Zhou, X. Y. Jing, P. F. Xie, W. H. Chen, T. Wang, H. Y. Xia and Z. J. Qin, FEMS Microbiol Lett, 2012, 333, 169-179.

64. D. G. Gibson, G. A. Benders, C. Andrews-Pfannkoch, E. A. Denisova, H. Baden-Tillson, J. Zaveri, T. B. Stockwell, A. Brownley, D. W. Thomas, M. A. Algire, C. Merryman, L. Young, V. N. Noskov, J. I. Glass, J. C. Venter, C. A. Hutchison, 3rd and H. O. Smith, Science, 2008, 319, 1215-1220.

65. C. A. Hutchison, 3rd, R. Y. Chuang, V. N. Noskov, N. AssadGarcia, T. J. Deerinck, M. H. Ellisman, J. Gill, K. Kannan, B. J. Karas, L. Ma, J. F. Pelletier, Z. Q. Qi, R. A. Richter, E. A. Strychalski, L. Sun, Y. Suzuki, B. Tsvetanova, K. S. Wise, H. O. Smith, J. I. Glass, C. Merryman, D. G. Gibson and J. C. Venter, Science, 2016, 351, aad6253.
66. J. E. Venetz, L. Del Medico, A. Wolfle, P. Schachle, Y. Bucher, D. Appert, F. Tschan, C. E. Flores-Tinoco, M. van Kooten, R. Guennoun, S. Deutsch, M. Christen and B. Christen, Proc Natl Acad Sci U S A, 2019, 116, 8070-8079.

67. R. C. Coates, B. P. Bowen, E. Oberortner, L. Thomashow, M. Hadjithomas, Z. Y. Zhao, J. Ke, L. Silva, K. Louie, G. Y. Wang, D. Robinson, A. Tarver, M. Hamilton, A. Lubbe, M. Feltcher, J. L. Dangl, A. Pati, D. Weller, T. R. Northen, J. F. Cheng, N. J. Mouncey, S. Deutsch and Y. Yoshikuni, J Ind Microbiol Biot, 2018, 45, 567-577.

68. P. Yakovchuk, E. Protozanova and M. D. Frank-Kamenetskii, Nucleic Acids Res, 2006, 34, 564-574.

69. S. Hwang, N. Lee, Y. Jeong, Y. Lee, W. Kim, S. Cho, B. O. Palsson and B. K. Cho, Nucleic Acids Res, 2019, 47, 6114-6129.

70. Y. X. Zhang, K. Perry, V. A. Vinci, K. Powell, W. P. Stemmer and S. B. del Cardayré, Nature, 2002, 415, 644-646.

71. H. S. Cho, J. C. Jo, C. H. Shin, N. Lee, J. S. Choi, B. K. Cho, J. H. Roe, C. W. Kim, H. J. Kwon and Y. J. Yoon, J Ind Microbiol Biotechnol, 2019, 46, 1205-1215.

72. P. Charusanti, N. L. Fong, H. Nagarajan, A. R. Pereira, H. J. Li, E. A. Abate, Y. Su, W. H. Gerwick and B. O. Palsson, PLoS One, 2012, 7, e33727.

73. A. M. Gehring, J. R. Nodwell, S. M. Beverley and R. Losick, Proc Natl Acad Sci U S A, 2000, 97, 9642-9647.

74. L. T. Fernandez-Martinez, R. Del Sol, M. C. Evans, S. Fielding, P. R. Herron, G. Chandra and P. J. Dyson, Antonie Van Leeuwenhoek, 2011, 99, 515-522.

75. Z. Xu, Y. Wang, K. F. Chater, H. Y. Ou, H. H. Xu, Z. Deng and M. Tao, Appl Environ Microbiol, 2017, 83.

76. F. Rousset, L. Cui, E. Siouve, C. Becavin, F. Depardieu and D. Bikard, PLoS Genet, 2018, 14, e1007749.

77. G. P. van Wezel and K. J. McDowall, Nat Prod Rep, 2011, 28, 1311-1333.

78. Y. Jeong, J. N. Kim, M. W. Kim, G. Bucca, S. Cho, Y. J. Yoon, B. G. Kim, J. H. Roe, S. C. Kim, C. P. Smith and B. K. Cho, Nat Commun, 2016, 7, 11605.

79. M. T. Alam, M. E. Merlo, S. Consortium, D. A. Hodgson, E. M. Wellington, E. Takano and R. Breitling, BMC Genomics, 2010, 11, 202.

80. N. J. Croucher and N. R. Thomson, Curr Opin Microbiol, 2010, 13, 619-624.

81. S. Zhao, W. P. Fung-Leung, A. Bittner, K. Ngo and X. Liu, PLoS One, 2014, 9, e78644.

82. K. Nieselt, F. Battke, A. Herbig, P. Bruheim, A. Wentzel, O. M. Jakobsen, H. Sletta, M. T. Alam, M. E. Merlo, J. Moore, W. A. Omara, E. R. Morrissey, M. A. Juarez-Hermosillo, A. RodriguezGarcia, M. Nentwich, L. Thomas, M. Iqbal, R. Legaie, W. H. Gaze, G. L. Challis, R. C. Jansen, L. Dijkhuizen, D. A. Rand, D. L. Wild, M. Bonin, J. Reuther, W. Wohlleben, M. C. Smith, N. J. Burroughs, J. F. Martin, D. A. Hodgson, E. Takano, R. Breitling, T. E. Ellingsen and E. M. Wellington, BMC Genomics, 2010, 11, 10.

83. L. Thomas, D. A. Hodgson, A. Wentzel, K. Nieselt, T. E. Ellingsen, J. Moore, E. R. Morrissey, R. Legaie, S. Consortium, W. Wohlleben, A. Rodriguez-Garcia, J. F. Martin, N. J. Burroughs, E. M. Wellington and M. C. Smith, Mol Cell Proteomics, 2012, 11, M111 013797.

84. B. Rioseras, M. T. Lopez-Garcia, P. Yague, J. Sanchez and A. Manteca, Bioresour Technol, 2014, 151, 191-198.

85. N. Zaburannyi, M. Rabyk, B. Ostash, V. Fedorenko and A. Luzhetskyy, BMC Genomics, 2014, 15, 97.

86. Y. Lee, N. Lee, Y. Jeong, S. Hwang, W. Kim, S. Cho, B. O. Palsson and B. K. Cho, Front Microbiol, 2019, 10, 2074. 
87. X. M. Zhu, X. X. Zhang, R. T. Cheng, H. L. Yu, R. S. Yuan, X. L. Bu, J. Xu, P. Ao, Y. C. Chen and M. J. Xu, R Soc Open Sci, 2019, 6, 190418.

88. J. Zhang, Z. He, J. Xu, S. Song, Q. Zhu, G. Wu, Y. Guan, X. Wu, R. Yue, Y. Wang, T. Yu, S. Hu, F. Lu and H. Zhang, Appl Microbiol Biotechnol, 2020, 104, 3459-3471.

89. S. Li, J. Wang, X. Li, S. Yin, W. Wang and K. Yang, Microb Cell Fact, 2015, 14, 172.

90. Y. Luo, L. Zhang, K. W. Barton and H. Zhao, ACS Synth Biol, 2015, 4, 1001-1010.

91. S. V. Sohoni, A. Fazio, C. T. Workman, I. Mijakovic and A. E. Lantz, PLoS One, 2014, 9, e99701.

92. M. W. Kim, B. R. Lee, S. You, E. J. Kim, J. N. Kim, E. Song, Y. H. Yang, D. Hwang and B. G. Kim, Appl Microbiol Biotechnol, 2018, 102, 3243-3253.

93. S. Li, J. Wang, W. Xiang, K. Yang, Z. Li and W. Wang, ACS Synth Biol, 2018, 7, 522-530.

94. G. Liao, Q. Liu and J. Xie, Microb Cell Fact, 2013, 12, 19.

95. G. Y. Tan, Y. Peng, C. Lu, L. Bai and J. J. Zhong, Metab Eng, 2015, 28, 74-81.

96. Q. Liot and P. Constant, Microbiologyopen, 2016, 5, 47-59.

97. A. J. Book, G. R. Lewin, B. R. McDonald, T. E. Takasuka, E. Wendt-Pienkowski, D. T. Doering, S. Suh, K. F. Raffa, B. G. Fox and C. R. Currie, PLoS Biol, 2016, 14, e1002475.

98. J. Koepff, H. Morschett, T. Busche, A. Winkler, J. Kalinowski, W. Wiechert and M. Oldiges, Biotechnol Prog, 2018, 34, 287-292.

99. S. E. Jones, L. Ho, C. A. Rees, J. E. Hill, J. R. Nodwell and M. A. Elliot, Elife, 2017, 6.

100. W. Daniels, J. Bouvin, T. Busche, C. Ruckert, K. Simoens, S. Karamanou, L. Van Mellaert, O. H. Friethjonsson, B. Nicolai, A. Economou, J. Kalinowski, J. Anne and K. Bernaerts, Microb Cell Fact, 2018, 17, 198.

101. R. Howlett, N. Read, A. Varghese, C. Kershaw, Y. Hancock and M. C. M. Smith, Microbiology, 2018, 164, 369-382

102. A. Dodd, D. Swanevelder, N. Zhou, D. Brady, J. E. Hallsworth and K. Rumbold, J Ind Microbiol Biotechnol, 2018, 45, 10831090.

103. M. J. Szafran, M. Gongerowska, T. Malecki, M. Elliot and D. Jakimowicz, Front Microbiol, 2019, 10, 1605.

104. L. Pan, X. Chen, K. Wang and Z. Mao, J Ind Microbiol Biotechnol, 2019, 46, 1781-1792.

105. L. Pinilla, L. F. Toro, E. Laing, J. F. Alzate and R. Rios-Estepa, Antibiotics (Basel), 2019, 8

106. O. T. Koshla, I. V. Rokytskyy, I. S. Ostash, T. Busche, J. Kalinowski, E. Mösker, R. D. Süssmuth, V. O. Fedorenko and B. O. Ostash, Cytology and Genetics, 2019, 53, 1-7.

107. C. Wang, J. Wang, J. Yuan, L. Jiang, X. Jiang, B. Yang, G. Zhao, B. Liu and D. Huang, Biotechnol Bioeng, 2019, 116, 3382-3395.

108. P. E. Sineli, D. S. Guerrero, A. Alvarez and J. S. Davila Costa, Appl Microbiol Biotechnol, 2019, 103, 5015-5022.

109. N. Lee, W. Kim, J. Chung, Y. Lee, S. Cho, K. S. Jang, S. C. Kim, B. Palsson and B. K. Cho, ISME J, 2020, 14, 1111-1124.

110. J. K. Schniete, R. Reunerman, L. Kerr, N. P. Tucker, L. S. Hunter, P. R. Herron and P. A. Hoskisson, Access Microbiology, 2020, 2.

111. S. Li, J. Ji, S. Hu and G. Chen, Bioprocess Biosyst Eng, 2020, 43, 1913-1921.

112. W. Shen, D. Wang, L. Wei and Y. Zhang, Appl Microbiol Biotechnol, 2020, 104, 4471-4482.

113. T. Arseneault, R. Roquigny, A. Novinscak, C. Goyer and M. Filion, Physiological and Molecular Plant Pathology, 2020, 110, 101480.

114. H. Li, J. Wei, J. Dong, Y. Li, Y. Li, Y. Chen and W. Guan, App/
Environ Microbiol, 2020, 86.

115. S. Panthee, N. Kito, T. Hayashi, T. Shimizu, J. Ishikawa, H. Hamamoto, H. Osada and S. Takahashi, Sci Rep, 2020, 10, 10230.

116. Z. W. Li, S. Liang, Y. Ke, J. J. Deng, M. S. Zhang, D. L. Lu, J. Z. Li and X. C. Luo, Commun Biol, 2020, 3, 191.

117. M. L. Gatewood, P. Bralley, M. R. Weil and G. H. Jones, J Bacteriol, 2012, 194, 2228-2237.

118. S. Dwarakanath, A. K. Chaplin, M. A. Hough, S. Rigali, E. Vijgenboom and J. A. Worrall, J Biol Chem, 2012, 287, 1783317847.

119. N. Naseer, J. A. Shapiro and M. Chander, PLoS One, 2014, 9, e106181.

120. S. Antoraz, S. Rico, H. Rodriguez, L. Sevillano, J. F. Alzate, R. I. Santamaria and M. Diaz, Front Microbiol, 2017, 8, 2444.

121. H. J. Cho, Y. S. Kwon, D. R. Kim, G. Cho, S. W. Hong, D. W. Bae and Y. S. Kwak, Microbiologyopen, 2017, 6.

122. P. Zhang, L. Wu, Y. Zhu, M. Liu, Y. Wang, G. Cao, X. L. Chen, M. Tao and X. Pang, Front Microbiol, 2017, 8, 2013.

123. Q. Liu, D. Pinto and T. Mascher, J Bacteriol, 2018, 200.

124. M. T. Lopez-Garcia, P. Yague, N. Gonzalez-Quinonez, B. Rioseras and A. Manteca, Front Microbiol, 2018, 9, 312.

125. S. Martin-Martin, A. Rodriguez-Garcia, F. Santos-Beneit, E. Franco-Dominguez, A. Sola-Landa and J. F. Martin, J Antibiot (Tokyo), 2017, 71, 113-122.

126. E. E. Arroyo-Perez, G. Gonzalez-Ceron, G. Soberon-Chavez, D. Georgellis and L. Servin-Gonzalez, Front Microbiol, 2019, 10, 1568.

127. J. Zhang, Q. Liang, Z. Xu, M. Cui, Q. Zhang, S. Abreu, M. David, C. Lejeune, P. Chaminade, M. J. Virolle and D. Xu, Front Microbiol, 2020, 11, 1399

128. C. Y. Lin, Y. Zhang, J. H. Wu, R. H. Xie, J. Qiao and G. R. Zhao, Microorganisms, 2020, 8.

129. C. Y. Lin, A. P. Pang, Y. Zhang, J. Qiao and G. R. Zhao, Microb Cell Fact, 2020, 19, 30.

130. J. Davies, G. B. Spiegelman and G. Yim, Curr Opin Microbiol, 2006, 9, 445-453.

131. D. Kong, X. Wang, J. Nie and G. Niu, Front Microbiol, 2019, 10, 2927.

132. M. Ishizuka, Y. Imai, K. Mukai, K. Shimono, R. Hamauzu, K. Ochi and T. Hosaka, Antonie Van Leeuwenhoek, 2018, 111, 705-716.

133. S. Shima, H. Matsuoka, T. Iwamoto and H. Sakai, J Antibiot (Tokyo), 1984, 37, 1449-1455.

134. P. Kahar, T. Iwata, J. Hiraki, E. Y. Park and M. Okabe, J Biosci Bioeng, 2001, 91, 190-194.

135. K. Ueda, S. Kawai, H. Ogawa, A. Kiyama, T. Kubota, H. Kawanobe and T. Beppu, J Antibiot (Tokyo), 2000, 53, 979-982.

136. J. Perez, J. Munoz-Dorado, A. F. Brana, L. J. Shimkets, L. Sevillano and R. I. Santamaria, Microb Biotechnol, 2011, 4, 175183.

137. J. Wakefield, H. M. Hassan, M. Jaspars, R. Ebel and M. E. Rateb, Front Microbiol, 2017, 8, 1284.

138. C. M. Sharma, S. Hoffmann, F. Darfeuille, J. Reignier, S. Findeiss, A. Sittka, S. Chabas, K. Reiche, J. Hackermuller, R. Reinhardt, P. F. Stadler and J. Vogel, Nature, 2010, 464, 250-255.

139. D. Dar, M. Shamir, J. R. Mellin, M. Koutero, N. Stern-Ginossar, P. Cossart and R. Sorek, Science, 2016, 352, aad9822.

140. J. T. Munnoch, M. T. Martinez, D. A. Svistunenko, J. C. Crack, N. E. Le Brun and M. I. Hutchings, Sci Rep, 2016, 6, 31597.

141. J. S. Bauer, S. Fillinger, K. Forstner, A. Herbig, A. C. Jones, K. Flinspach, C. Sharma, H. Gross, K. Nieselt and A. K. Apel, RNA Biol, 2017, 14, 1617-1626. 
142. M. P. Vockenhuber, C. M. Sharma, M. G. Statt, D. Schmidt, Z. $\mathrm{Xu}, \mathrm{S}$. Dietrich, H. Liesegang, D. H. Mathews and B. Suess, RNA Biol, 2011, 8, 468-477.

143. D. A. Romero, A. H. Hasan, Y. F. Lin, L. Kime, O. Ruiz-Larrabeiti, M. Urem, G. Bucca, L. Mamanova, E. E. Laing, G. P. van Wezel, C. P. Smith, V. R. Kaberdin and K. J. McDowall, Mol Microbiol, 2014, 94, 963-987.

144. A. Staron, H. J. Sofia, S. Dietrich, L. E. Ulrich, H. Liesegang and T. Mascher, Mol Microbiol, 2009, 74, 557-581.

145. J. S. Yi, M. W. Kim, M. Kim, Y. Jeong, E. J. Kim, B. K. Cho and B. G. Kim, ACS Synth Biol, 2017, 6, 555-565.

146. D. Dar and R. Sorek, Nucleic Acids Res, 2018, 46, 6797-6805.

147. I. Gusarov and E. Nudler, Cell, 2001, 107, 437-449.

148. N. Lee, S. Hwang, Y. Lee, S. Cho, B. Palsson and B. K. Cho, J Microbiol Biotechnol, 2019, 29, 667-686.

149. D. Sun, C. Liu, J. Zhu and W. Liu, Front Microbiol, 2017, 8, 2546.

150. A. Romero-Rodriguez, I. Robledo-Casados and S. Sanchez, Biochim Biophys Acta, 2015, 1849, 1017-1039.

151. K. F. Chater and G. Chandra, FEMS Microbiol Rev, 2006, 30, 651-672.

152. K. Flardh and M. J. Buttner, Nat Rev Microbiol, 2009, 7, 36-49.

153. G. Liu, K. F. Chater, G. Chandra, G. Niu and H. Tan, Microbiol Mol Biol Rev, 2013, 77, 112-143.

154. C. D. den Hengst, N. T. Tran, M. J. Bibb, G. Chandra, B. K. Leskiw and M. J. Buttner, Mol Microbiol, 2010, 78, 361-379.

155. J. G. Kang, M. Y. Hahn, A. Ishihama and J. H. Roe, Nucleic Acids Res, 1997, 25, 2566-2573.

156. K. Smidova, A. Zikova, J. Pospisil, M. Schwarz, J. Bobek and J. Vohradsky, Nucleic Acids Res, 2019, 47, 621-633.

157. K. A. Gallagher, M. A. Schumacher, M. J. Bush, M. J. Bibb, G. Chandra, N. A. Holmes, W. Zeng, M. Henderson, H. Zhang, K. C. Findlay, R. G. Brennan and M. J. Buttner, Mol Cell, 2020, 77, 586-599 e586.

158. B. Bilyk, S. Kim, A. Fazal, T. A. Baker and R. F. Seipke, mSphere, 2020, 5.

159. S. P. Liu, P. Yu, P. H. Yuan, Z. X. Zhou, Q. T. Bu, X. M. Mao and Y. Q. Li, Appl Microbiol Biotechnol, 2015, 99, 2715-2726.

160. R. Chauhan, J. Ravi, P. Datta, T. Chen, D. Schnappinger, K. E. Bassler, G. Balazsi and M. L. Gennaro, Nat Commun, 2016, 7, 11062.

161. N. T. Tran, X. Huang, H. J. Hong, M. J. Bush, G. Chandra, D. Pinto, M. J. Bibb, M. I. Hutchings, T. Mascher and M. J. Buttner, Mol Microbiol, 2019, 112, 461-481.

162. A. Higo, H. Hara, S. Horinouchi and Y. Ohnishi, DNA Res, 2012, 19, 259-273.

163. M. M. Al-Bassam, M. J. Bibb, M. J. Bush, G. Chandra and M. J. Buttner, PLoS Genet, 2014, 10, e1004554.

164. P. G. Becher, V. Verschut, M. J. Bibb, M. J. Bush, B. P. Molnar, E. Barane, M. M. Al-Bassam, G. Chandra, L. Song, G. L. Challis, M. J. Buttner and K. Flardh, Nat Microbiol, 2020, 5, 821-829.

165. N. F. Som, D. Heine, N. Holmes, F. Knowles, G. Chandra, R. F. Seipke, P. A. Hoskisson, B. Wilkinson and M. I. Hutchings, Microbiology, 2017, 163, 1415-1419.

166. N. F. Som, D. Heine, N. A. Holmes, J. T. Munnoch, G. Chandra, R. F. Seipke, P. A. Hoskisson, B. Wilkinson and M. I. Hutchings, Front Microbiol, 2017, 8, 1145.

167. X. Li, J. Wang, S. Li, J. Ji, W. Wang and K. Yang, Sci Rep, 2015, 5, 14831

168. M. Liu, P. Zhang, Y. Zhu, T. Lu, Y. Wang, G. Cao, M. Shi, X. L. Chen, M. Tao and X. Pang, Appl Environ Microbiol, 2019, 85.

169. T. C. McLean, P. A. Hoskisson and R. F. Seipke, mSphere, 2016, 1.
170. J. Fu, R. Qin, G. Zong, C. Liu, N. Kang, C. Zhong and G. Cao, Front Microbiol, 2019, 10, 244.

171. E. J. Gehrke, X. Zhang, S. M. Pimentel-Elardo, A. R. Johnson, C. A. Rees, S. E. Jones, Hindra, S. S. Gehrke, S. Turvey, S. Boursalie, J. E. Hill, E. E. Carlson, J. R. Nodwell and M. A. Elliot, Elife, 2019, 8.

172. M. J. Bush, G. Chandra, M. M. Al-Bassam, K. C. Findlay and M. J. Buttner, mBio, 2019, 10, e02812-02818.

173. M. J. Bush, M. J. Bibb, G. Chandra, K. C. Findlay and M. J. Buttner, mBio, 2013, 4, e00684-00613.

174. M. J. Bush, G. Chandra, M. J. Bibb, K. C. Findlay and M. J. Buttner, mBio, 2016, 7, e00523-00516.

175. N. Tschowri, M. A. Schumacher, S. Schlimpert, N. B. Chinnam, K. C. Findlay, R. G. Brennan and M. J. Buttner, Cell, 2014, 158, 1136-1147.

176. M. A. Schumacher, W. Zeng, K. C. Findlay, M. J. Buttner, R. G. Brennan and N. Tschowri, Nucleic Acids Res, 2017, 45, 69236933.

177. M. J. Bush, G. Chandra, K. C. Findlay and M. J. Buttner, Mol Microbiol, 2017, 104, 700-711.

178. J. N. Kim, Y. Jeong, J. S. Yoo, J. H. Roe, B. K. Cho and B. G. Kim, BMC Genomics, 2015, 16, 116.

179. J. H. Lee, J. S. Yoo, Y. Kim, J. S. Kim, E. J. Lee and J. H. Roe, mBio, 2020, 11

180. J. C. Crack, J. Munnoch, E. L. Dodd, F. Knowles, M. M. Al Bassam, S. Kamali, A. A. Holland, S. P. Cramer, C. J. Hamilton, M. K. Johnson, A. J. Thomson, M. I. Hutchings and N. E. Le Brun, J Biol Chem, 2015, 290, 12689-12704.

181. Y. Chen, M. J. Smanski and B. Shen, Appl Microbiol Biotechnol, 2010, 86, 19-25.

182. S. Hackl and A. Bechthold, Arch Pharm (Weinheim), 2015, 348, 455-462.

183. E. Takano, M. Tao, F. Long, M. J. Bibb, L. Wang, W. Li, M. J. Buttner, M. J. Bibb, Z. X. Deng and K. F. Chater, Mol Microbiol, 2003, 50, 475-486.

184. M. A. Fernandez-Moreno, J. L. Caballero, D. A. Hopwood and F. Malpartida, Cell, 1991, 66, 769-780.

185. A. Higo, S. Horinouchi and Y. Ohnishi, Mol Microbiol, 2011, 81, 1607-1622.

186. A. Hesketh, W. J. Chen, J. Ryding, S. Chang and M. Bibb, Genome Biol, 2007, 8, R161.

187. D. O. Klymyshin, O. M. Stephanyshyn and V. O. Fedorenko, Cytology and Genetics, 2016, 50, 134-142.

188. C. Condon and H. Putzer, Nucleic Acids Res, 2002, 30, 53395346.

189. S. E. Jones, V. Leong, J. Ortega and M. A. Elliot, J Bacteriol, 2014, 196, 4253-4267.

190. A. V. Sherwood, F. J. Grundy and T. M. Henkin, Proc Natl Acad Sci U S A, 2015, 112, 1113-1118.

191. I. Borovok, B. Gorovitz, R. Schreiber, Y. Aharonowitz and G. Cohen, J Bacteriol, 2006, 188, 2512-2520.

192. N. T. Ingolia, S. Ghaemmaghami, J. R. Newman and J. S. Weissman, Science, 2009, 324, 218-223.

193. W. Kim, S. Hwang, N. Lee, Y. Lee, S. Cho, B. Palsson and B. K. Cho, Sci Data, 2020, 7, 138.

194. F. Mohammad, R. Green and A. R. Buskirk, Elife, 2019, 8.

195. D. R. Gelsinger, E. Dallon, R. Reddy, F. Mohammad, A. R. Buskirk and J. DiRuggiero, Nucleic Acids Res, 2020, 48, 52015216.

196. A. B. Arpat, A. Liechti, M. De Matos, R. Dreos, P. Janich and D. Gatfield, Genome Res, 2020, 30, 985-999.

197. P. Han, Y. Shichino, T. Schneider-Poetsch, M. Mito, S. 
Hashimoto, T. Udagawa, K. Kohno, M. Yoshida, Y. Mishima, T. Inada and S. Iwasaki, Cell Rep, 2020, 31, 107610.

198. K. P. Jayapal, S. Sui, R. J. Philp, Y. J. Kok, M. G. Yap, T. J. Griffin and W. S. Hu, J Proteome Res, 2010, 9, 2087-2097.

199. J. Pospisil, D. Strunin, A. Zikova, M. Hubalek and J. Vohradsky, Proteomics, 2020, 20, e2000032.

200. K. P. Jayapal, R. J. Philp, Y. J. Kok, M. G. Yap, D. H. Sherman, T. J. Griffin and W. S. Hu, PLoS One, 2008, 3, e2097.

201. Y. Zhang, B. R. Fonslow, B. Shan, M. C. Baek and J. R. Yates, 3rd, Chem Rev, 2013, 113, 2343-2394.

202. A. Manteca, J. Sanchez, H. R. Jung, V. Schwammle and O. N. Jensen, Mol Cell Proteomics, 2010, 9, 1423-1436.

203. E. Palazzotto, G. Renzone, P. Fontana, L. Botta, A. Scaloni, A. M. Puglia and G. Gallo, Appl Microbiol Biotechnol, 2015, 99, 10177-10189.

204. Y. J. Kim, M. H. Moon, J. Y. Song, C. P. Smith, S. K. Hong and Y. K. Chang, BMC Genomics, 2008, 9, 604.

205. Y. J. Kim, J. Y. Song, M. H. Moon, C. P. Smith, S. K. Hong and Y. K. Chang, Appl Microbiol Biotechnol, 2007, 76, 1119-1130.

206. J.-S. Cheng, X.-M. Lv and Y.-J. Yuan, BIOTECHNOL BIOPROC E, 2012, 17, 997-1007.

207. I. Santamarta, M. T. Lopez-Garcia, A. Kurt, N. Nardiz, R. AlvarezAlvarez, R. Perez-Redondo, J. F. Martin and P. Liras, Mol Microbiol, 2011, 81, 968-981.

208. N. L. Ferguson, L. Pena-Castillo, M. A. Moore, D. R. Bignell and K. Tahlan, J Ind Microbiol Biotechnol, 2016, 43, 537-555.

209. P. Le Marechal, P. Decottignies, C. H. Marchand, J. Degrouard, D. Jaillard, T. Dulermo, M. Froissard, A. Smirnov, V. Chapuis and M. J. Virolle, Appl Environ Microbiol, 2013, 79, 5907-5917.

210. C. Wang, X. Long, X. Mao, H. Dong, L. Xu and Y. Li, Microbiol Res, 2010, 165, 221-231.

211. S. Okamoto, A. Lezhava, T. Hosaka, Y. Okamoto-Hosoya and K. Ochi, J Bacteriol, 2003, 185, 601-609.

212. E. Song, S. Malla, Y. H. Yang, K. Lee, E. J. Kim, H. C. Lee, J. K. Sohng, M. K. Oh and B. G. Kim, J Ind Microbiol Biotechnol, 2011, 38, 1245-1253.

213. Y. Zhao and O. N. Jensen, Proteomics, 2009, 9, 4632-4641.

214. Y. Ishigaki, G. Akanuma, M. Yoshida, S. Horinouchi, S. Kosono and Y. Ohnishi, J Proteomics, 2017, 155, 63-72.

215. A. Manteca, J. Ye, J. Sanchez and O. N. Jensen, J Proteome Res, 2011, 10, 5481-5492.

216. J. L. Parker, A. M. Jones, L. Serazetdinova, G. Saalbach, M. J. Bibb and M. J. Naldrett, Proteomics, 2010, 10, 2486-2497.

217. G. Liao, L. Xie, X. Li, Z. Cheng and J. Xie, J Proteomics, 2014, 106, 260-269.

218. B. Rioseras, P. V. Shliaha, V. Gorshkov, P. Yague, M. T. LopezGarcia, N. Gonzalez-Quinonez, S. Kovalchuk, A. RogowskaWrzesinska, O. N. Jensen and A. Manteca, Mol Cell Proteomics, 2018, 17, 1591-1611.

219. C. F. Sun, W. F. Xu, Q. W. Zhao, S. Luo, X. A. Chen, Y. Q. Li and X. M. Mao, Commun Biol, 2020, 3, 192.

220. H. Boubakri, N. Seghezzi, M. Duchateau, M. Gominet, O. Kofronova, O. Benada, P. Mazodier and J. L. Pernodet, J Bacteriol, 2015, 197, 3388-3399.

221. C. Du and G. P. van Wezel, Proteomics, 2018, 18, e1700332.

222. J. Gubbens, H. Zhu, G. Girard, L. Song, B. I. Florea, P. Aston, K. Ichinose, D. V. Filippov, Y. H. Choi, H. S. Overkleeft, G. L. Challis and G. P. van Wezel, Chem Biol, 2014, 21, 707-718.

223. C. Wu, C. Du, J. Gubbens, Y. H. Choi and G. P. van Wezel, J Nat Prod, 2015, 78, 2355-2363.

224. J. Gubbens, C. Wu, H. Zhu, D. V. Filippov, B. I. Florea, S. Rigali, H. S. Overkleeft and G. P. van Wezel, ACS Chem Biol, 2017, 12,
2756-2766.

225. P. C. Dorrestein, S. B. Bumpus, C. T. Calderone, S. GarneauTsodikova, Z. D. Aron, P. D. Straight, R. Kolter, C. T. Walsh and N. L. Kelleher, Biochemistry, 2006, 45, 12756-12766.

226. S. B. Bumpus, B. S. Evans, P. M. Thomas, I. Ntai and N. L. Kelleher, Nat Biotechnol, 2009, 27, 951-956.

227. Y. Chen, M. Unger, I. Ntai, R. A. McClure, J. C. Albright, R. J. Thomson and N. L. Kelleher, Medchemcomm, 2013, 4, 233238.

228. J. C. Albright, A. W. Goering, J. R. Doroghazi, W. W. Metcalf and N. L. Kelleher, J Ind Microbiol Biotechnol, 2014, 41, 451-459.

229. J. L. Meier, S. Niessen, H. S. Hoover, T. L. Foley, B. F. Cravatt and M. D. Burkart, ACS Chem Biol, 2009, 4, 948-957.

230. A. Hagen, S. Poust, T. Rond, J. L. Fortman, L. Katz, C. J. Petzold and J. D. Keasling, ACS Synth Biol, 2016, 5, 21-27.

231. R. D. Kersten, Y. L. Yang, Y. Xu, P. Cimermancic, S. J. Nam, W. Fenical, M. A. Fischbach, B. S. Moore and P. C. Dorrestein, Nat Chem Biol, 2011, 7, 794-802.

232. W. T. Liu, R. D. Kersten, Y. L. Yang, B. S. Moore and P. C. Dorrestein, J Am Chem Soc, 2011, 133, 18010-18013.

233. M. E. Rateb, Y. Zhai, E. Ehrner, C. M. Rath, X. Wang, J. Tabudravu, R. Ebel, M. Bibb, K. Kyeremeh, P. C. Dorrestein, K. Hong, M. Jaspars and H. Deng, Org Biomol Chem, 2015, 13, 9585-9592.

234. W. T. Liu, A. Lamsa, W. R. Wong, P. D. Boudreau, R. Kersten, Y. Peng, W. J. Moree, B. M. Duggan, B. S. Moore, W. H. Gerwick, R. G. Linington, K. Pogliano and P. C. Dorrestein, J Antibiot (Tokyo), 2014, 67, 99-104.

235. S. Hwang, N. Lee, S. Cho, B. Palsson and B. K. Cho, Front Mol Biosci, 2020, 7, 87.

236. J. Zhao, G. Wang, J. Chu and Y. Zhuang, World J Microbiol Biotechnol, 2019, 36, 1.

237. C. Wu, H. K. Kim, G. P. van Wezel and Y. H. Choi, Drug Discov Today Technol, 2015, 13, 11-17.

238. R. Sinha, B. Sharma, A. K. Dangi and P. Shukla, World J Microbiol Biotechnol, 2019, 35, 166.

239. P. S. Gromski, H. Muhamadali, D. I. Ellis, Y. Xu, E. Correa, M. L. Turner and R. Goodacre, Anal Chim Acta, 2015, 879, 10-23.

240. A. T. Aron, E. C. Gentry, K. L. McPhail, L. F. Nothias, M. NothiasEsposito, A. Bouslimani, D. Petras, J. M. Gauglitz, N. Sikora, F. Vargas, J. J. J. van der Hooft, M. Ernst, K. B. Kang, C. M. Aceves, A. M. Caraballo-Rodriguez, I. Koester, K. C. Weldon, S. Bertrand, C. Roullier, K. Sun, R. M. Tehan, P. C. Boya, M. H. Christian, M. Gutierrez, A. M. Ulloa, J. A. Tejeda Mora, R. Mojica-Flores, J. Lakey-Beitia, V. Vasquez-Chaves, Y. Zhang, A. I. Calderon, N. Tayler, R. A. Keyzers, F. Tugizimana, N. Ndlovu, A. A. Aksenov, A. K. Jarmusch, R. Schmid, A. W. Truman, N. Bandeira, M. Wang and P. C. Dorrestein, Nat Protoc, 2020, 15, 1954-1991.

241. M. Wang, J. J. Carver, V. V. Phelan, L. M. Sanchez, N. Garg, Y. Peng, D. D. Nguyen, J. Watrous, C. A. Kapono, T. LuzzattoKnaan, C. Porto, A. Bouslimani, A. V. Melnik, M. J. Meehan, W. T. Liu, M. Crusemann, P. D. Boudreau, E. Esquenazi, M. Sandoval-Calderon, R. D. Kersten, L. A. Pace, R. A. Quinn, K. R. Duncan, C. C. Hsu, D. J. Floros, R. G. Gavilan, K. Kleigrewe, T. Northen, R. J. Dutton, D. Parrot, E. E. Carlson, B. Aigle, C. F. Michelsen, L. Jelsbak, C. Sohlenkamp, P. Pevzner, A. Edlund, J. McLean, J. Piel, B. T. Murphy, L. Gerwick, C. C. Liaw, Y. L. Yang, H. U. Humpf, M. Maansson, R. A. Keyzers, A. C. Sims, A. R. Johnson, A. M. Sidebottom, B. E. Sedio, A. Klitgaard, C. B. Larson, C. A. B. P, D. Torres-Mendoza, D. J. Gonzalez, D. B. Silva, L. M. Marques, D. P. Demarque, E. Pociute, E. C. O'Neill, E. 
Briand, E. J. N. Helfrich, E. A. Granatosky, E. Glukhov, F. Ryffel, H. Houson, H. Mohimani, J. J. Kharbush, Y. Zeng, J. A. Vorholt, K. L. Kurita, P. Charusanti, K. L. McPhail, K. F. Nielsen, L. Vuong, M. Elfeki, M. F. Traxler, N. Engene, N. Koyama, O. B. Vining, R. Baric, R. R. Silva, S. J. Mascuch, S. Tomasi, S. Jenkins, V. Macherla, T. Hoffman, V. Agarwal, P. G. Williams, J. Dai, R. Neupane, J. Gurr, A. M. C. Rodriguez, A. Lamsa, C. Zhang, K. Dorrestein, B. M. Duggan, J. Almaliti, P. M. Allard, P. Phapale, L. F. Nothias, T. Alexandrov, M. Litaudon, J. L. Wolfender, J. E. Kyle, T. O. Metz, T. Peryea, D. T. Nguyen, D. VanLeer, P. Shinn, A. Jadhav, R. Muller, K. M. Waters, W. Shi, X. Liu, L. Zhang, R. Knight, P. R. Jensen, B. O. Palsson, K. Pogliano, R. G. Linington, M. Gutierrez, N. P. Lopes, W. H. Gerwick, B. S. Moore, P. C. Dorrestein and N. Bandeira, Nat Biotechnol, 2016, 34, 828-837.

242. K. A. Hollywood, K. Schmidt, E. Takano and R. Breitling, Curr Opin Biotechnol, 2018, 54, 114-120.

243. A. Amara, E. Takano and R. Breitling, BMC Genomics, 2018, 19, 519.

244. D. Huang, S. Li, M. Xia, J. Wen and X. Jia, Microb Cell Fact, 2013, 12, 52.

245. H. Qi, S. Zhao, H. Fu, J. Wen and X. Jia, Biochemical Engineering Journal, 2014, 91, 186-195.

246. B. Zhang, Y. T. Zhou, S. X. Jiang, Y. H. Zhang, K. Huang, Z. Q. Liu and Y. G. Zheng, Microb Cell Fact, 2020, 19, 18.

247. P. Tian, P. Cao, D. Hu, D. Wang, J. Zhang, L. Wang, Y. Zhu and Q. Gao, J Ind Microbiol Biotechnol, 2017, 44, 595-604.

248. M. Sandoval-Calderon, D. D. Nguyen, C. A. Kapono, P. Herron, P. C. Dorrestein and C. Sohlenkamp, Front Microbiol, 2015, 6, 1465.

249. C. Wang, D. Huang and S. Liang, Appl Microbiol Biotechnol, 2018, 102, 7541-7553.

250. J. Wang, H. Liu, D. Huang, L. Jin, C. Wang and J. Wen, Appl Microbiol Biotechnol, 2017, 101, 2447-2465.

251. X. Liu, X. Sun, T. Wang, X. Zhang, X. Tian, Y. Zhuang and J. Chu, Bioprocess Biosyst Eng, 2018, 41, 1743-1755.

252. C. A. Boya P, M. H. Christian, H. Fernández-Marín and M. Gutiérrez, Nat Prod Commun, 2019, 14, 63-66.

253. P. C. Boya, H. Fernandez-Marin, L. C. Mejia, C. Spadafora, P. C. Dorrestein and M. Gutierrez, Sci Rep, 2017, 7, 5604.

254. X. Cai, R. Teta, C. Kohlhaas, M. Crusemann, R. Ueoka, A. Mangoni, M. F. Freeman and J. Piel, Chem Biol, 2013, 20, 839846.

255. W. Wang, S. Li, Z. Li, J. Zhang, K. Fan, G. Tan, G. Ai, S. M. Lam, G. Shui, Z. Yang, H. Lu, P. Jin, Y. Li, X. Chen, X. Xia, X. Liu, H. K. Dannelly, C. Yang, Y. Yang, S. Zhang, G. Alterovitz, W. Xiang and L. Zhang, Nat Biotechnol, 2020, 38, 76-83.

256. G. Cox, A. Sieron, A. M. King, G. De Pascale, A. C. Pawlowski, K. Koteva and G. D. Wright, Cell Chem Biol, 2017, 24, 98-109.

257. R. Breitling, A. Ceniceros, A. Jankevics and E. Takano, Metabolites, 2013, 3, 1076-1083.

258. N. V. Machushynets, C. Wu, S. S. Elsayed, T. Hankemeier and G. P. van Wezel, J Ind Microbiol Biotechnol, 2019, 46, 483-492.

259. A. M. Sidebottom, A. R. Johnson, J. A. Karty, D. J. Trader and E. E. Carlson, ACS Chem Biol, 2013, 8, 2009-2016.

260. Y. Shi, C. Pan, B. N. Auckloo, X. Chen, C. A. Chen, K. Wang, X. Wu, Y. Ye and B. Wu, Appl Microbiol Biotechnol, 2017, 101, 1395-1408.

261. N. Akhter, Y. Liu, B. N. Auckloo, Y. Shi, K. Wang, J. Chen, X. Wu and B. Wu, Mar Drugs, 2018, 16.

262. F. Xu, Y. Wu, C. Zhang, K. M. Davis, K. Moon, L. B. Bushin and M. R. Seyedsayamdost, Nat Chem Biol, 2019, 15, 161-168.

263. A. M. Caraballo-Rodriguez, P. C. Dorrestein and M. T. Pupo, Sci
Rep, 2017, 7, 5373.

264. R. Sugiyama, S. Nishimura, T. Ozaki, S. Asamizu, H. Onaka and H. Kakeya, Org Lett, 2015, 17, 1918-1921.

265. C. Wu, H. Zhu, G. P. van Wezel and Y. H. Choi, Metabolomics, 2016, 12, 90.

266. L. Y. Chen, H. T. Cui, C. Su, F. W. Bai and X. Q. Zhao, PeerJ, 2019, 7, e6122.

267. N. M. Ishaque, I. Burgsdorf, J. J. Limlingan Malit, S. Saha, R. Teta, D. Ewe, K. Kannabiran, P. Hrouzek, L. Steindler, V. Costantino and K. Saurav, Microorganisms, 2020, 8.

268. A. Bauermeister, F. Pereira, I. R. Grilo, C. C. Godinho, M. Paulino, V. Almeida, L. Gobbo-Neto, A. Prieto-Davo, R. G. Sobral, N. P. Lopes and S. P. Gaudencio, Environ Microbiol, 2019, 21, 1099-1112.

269. J. C. Navarro-Munoz, N. Selem-Mojica, M. W. Mullowney, S. A. Kautsar, J. H. Tryon, E. I. Parkinson, E. L. C. De Los Santos, M. Yeong, P. Cruz-Morales, S. Abubucker, A. Roeters, W. Lokhorst, A. Fernandez-Guerra, L. T. D. Cappelini, A. W. Goering, R. J. Thomson, W. W. Metcalf, N. L. Kelleher, F. Barona-Gomez and M. H. Medema, Nat Chem Biol, 2020, 16, 60-68.

270. M. F. Traxler, J. D. Watrous, T. Alexandrov, P. C. Dorrestein and R. Kolter, mBio, 2013, 4, e00459-00413.

271. J. Li, H. Wang and M. C. Jewett, Biochemical Engineering Journal, 2018, 130, 29-33.

272. M. Myronovskyi, E. Welle, V. Fedorenko and A. Luzhetskyy, Appl Environ Microbiol, 2011, 77, 5370-5383.

273. R. Knirschova, R. Novakova, E. Mingyar, C. Bekeova, D. Homerova and J. Kormanec, J Microbiol Methods, 2015, 113, 1 3.

274. J. Sun, G. H. Kelemen, J. M. Fernández-Abalos and M. J. Bibb, Microbiology, 1999, 145, 2221-2227.

275. R. M. Phelan, D. Sachs, S. J. Petkiewicz, J. F. Barajas, J. M. BlakeHedges, M. G. Thompson, A. Reider Apel, B. J. Rasor, L. Katz and J. D. Keasling, ACS Synth Biol, 2017, 6, 159-166.

276. C. Bai, Y. Zhang, X. Zhao, Y. Hu, S. Xiang, J. Miao, C. Lou and L. Zhang, Proc Natl Acad Sci U S A, 2015, 112, 12181-12186.

277. C. M. Kasey, M. Zerrad, Y. Li, T. A. Cropp and G. J. Williams, ACS Synth Biol, 2018, 7, 227-239.

278. Y. Q. Sun, T. Busche, C. Ruckert, C. Paulus, Y. Rebets, R. Novakova, J. Kalinowski, A. Luzhetskyy, J. Kormanec, O. N. Sekurova and S. B. Zotchev, ACS Synth Biol, 2017, 6, 1026-1033.

279. J. Tan, A. V. Sastry, K. S. Fremming, S. P. Bjorn, A. Hoffmeyer, S. Seo, B. G. Voldborg and B. O. Palsson, Metab Eng, 2020, 61, 360-368.

280. K. R. Choi, W. D. Jang, D. Yang, J. S. Cho, D. Park and S. Y. Lee, Trends Biotechnol, 2019, 37, 817-837. 